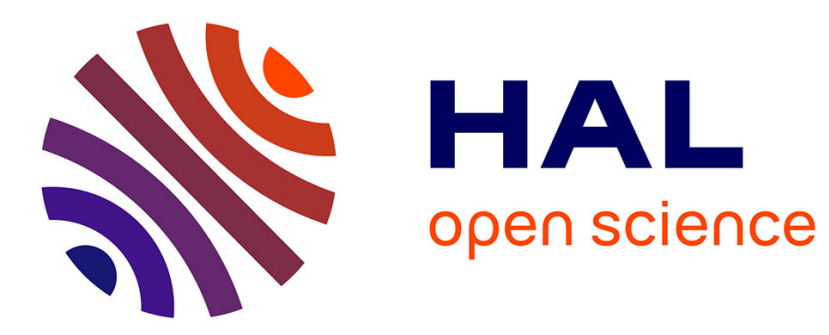

\title{
Fictitious domain method and separated representations for the solution of boundary value problems on uncertain parameterized domains
}

\author{
Anthony Nouy, Mathilde Chevreuil, Elias Safatly
}

\section{- To cite this version:}

Anthony Nouy, Mathilde Chevreuil, Elias Safatly. Fictitious domain method and separated representations for the solution of boundary value problems on uncertain parameterized domains. Computer Methods in Applied Mechanics and Engineering, 2011, 200 (45-46), pp.3066-3082. 10.1016/j.cma.2011.07.002 . hal-00662564

\section{HAL Id: hal-00662564 https://hal.science/hal-00662564}

Submitted on 24 Jan 2012

HAL is a multi-disciplinary open access archive for the deposit and dissemination of scientific research documents, whether they are published or not. The documents may come from teaching and research institutions in France or abroad, or from public or private research centers.
L'archive ouverte pluridisciplinaire HAL, est destinée au dépôt et à la diffusion de documents scientifiques de niveau recherche, publiés ou non, émanant des établissements d'enseignement et de recherche français ou étrangers, des laboratoires publics ou privés. 


\title{
Fictitious domain method and separated representations for the solution of boundary value problems on uncertain parameterized domains $^{1}$
}

\author{
A. Nouy*, M. Chevreuil and E. Safatly \\ LUNAM Université, GeM, UMR CNRS 6183, Centrale Nantes, Université de \\ Nantes, 1 rue de la Noë, BP 92101, 44321 Nantes Cedex 3, France.
}

\begin{abstract}
A tensor-based method is proposed for the solution of partial differential equations defined on uncertain parameterized domains. It provides an accurate solution which is explicit with respect to parameters defining the shape of the domain, thus allowing efficient a posteriori probabilistic or parametric analyses. In the proposed method, a fictitious domain approach is first adopted for the reformulation of the parametric problem on a fixed domain, yielding a weak formulation in a tensor product space (product of space functions and parametric functions). The paper is limited to the case of Neumann conditions on uncertain parts of the boundary. The Proper Generalized Decomposition method is then introduced for the construction of a tensor product approximation (separated representation) of the solution. It can be seen as an a priori model reduction technique which automatically captures reduced bases of space functions and parametric functions which are optimal for the representation of the solution. This tensor-based method is made computationally tractable by introducing separated representations of variational forms, resulting from separated representations of the parameterized indicator function of the uncertain domain. For this purpose, a method is proposed for the construction of a constrained tensor product approximation which preserves positivity and therefore ensures well-posedness of problems associated with approximate indicator functions. Moreover, a regularization of the geometry is introduced to speed up the convergence of these tensor product approximations.
\end{abstract}

Key words: Uncertainty quantification, Proper Generalized Decomposition, Tensor Product approximation, Random domain, Fictitious domain method, Spectral stochastic methods 


\section{Introduction}

Uncertainty quantification has become a critical issue in computational and prediction science. Uncertainty may reflect inherent variabilities in physical systems which have to be incorporated in mathematical models, or some level of ignorance that yields an imprecise or incomplete characterization of a model. When adopting a probabilistic modeling of uncertainties, their impact on a model output may be classically evaluated by means of classical sampling techniques if one is interested in probabilistic or statistical quantities of interest. In the last two decades, a growing attention has been given to an alternative functional view of uncertainty quantification, where uncertain (random) quantities are seen as functionals of parameters characterizing the input uncertainties. This functional view, combined with approximation theory and numerical analysis, has led to the development of a family of numerical methods, called spectral stochastic methods, for the propagation of uncertainties through a model, yielding a complete characterization of uncertain model outputs (see recent reviews $[19,30,14]$ and $[13])$. The introduction of model uncertainty may also be required for different purposes such as model design, identification or optimization, where one is interested in the quantification of model outputs for a certain range of input parameters. In this context of parametric analyses, spectral methods provide an explicit representation of the output with respect to input parameters, thus allowing a posteriori parametric analyses. Therefore, they constitute efficient alternatives to traditional methods that require many evaluations of the initial model for particular values of input parameters (e.g. corresponding to an experimental design, iterates of an optimization procedure...).

Spectral methods for uncertainty quantification have been successfully applied to many problems in science and engineering. In particular, many works have considered the uncertainty propagation through models involving partial differential equations (PDEs) with uncertain operators and source terms (see e.g. $[9,4,15,28])$. A few works have been recently devoted to numerical methods for PDEs defined on uncertain domains $[31,29,5,23,2,16,10,22]$. The explicit characterization of output quantities with respect to input shape parameters is of great interest in various analyses: impact of random perturbations of a shape, shape optimization in model design, inverse analysis in non destructive testing (location of a defect)... The above mentioned works start with a reformulation of the problem on a fixed deterministic domain. In $[31,29,16]$, a random mapping maps the random domain into a deterministic domain, thus transforming

\footnotetext{
* Corresponding author. Tel: +33-2-40-37-16-76

Email address: anthony.nouy@ec-nantes.fr (A. Nouy).

1 This work is supported by the French National Research Agency (grant ANR2010-COSI-006-01).
} 
a PDE defined on an uncertain domain into a PDE defined on a fixed domain with uncertain operator and right-hand-side depending on the mapping and its derivatives. In [5,23], fictitious domain methods are introduced and consist in embedding the uncertain domain into a fixed domain, the geometry being characterized with a level-set technique or a mapping technique.

\section{Contribution of this paper}

In this paper, we present an efficient method for the numerical solution of PDEs defined on uncertain parameterized domains $\Omega(\xi)$, with $\xi \in \Xi$ being parameters (eventually random), which combines a fictitious domain approach and a tensor-based method, namely the Proper Generalized Decomposition, for the construction of optimal separated representations of the solution. For the proposed tensor-based method to be computationally tractable, additional technical ingredients are introduced in order to recast the problem in a suitable tensor format. These ingredients consist of specific treatments of the random geometry, more precisely of the indicator function representing the random domain. The impact of these approximations of the geometry are carefully analyzed.

As a model example, we consider a simple diffusion equation $-\Delta u=f$ defined on $\Omega(\xi)$. The paper is limited to the case of Neumann conditions on uncertain parts of the boundary.

A fictitious domain approach is first adopted for the reformulation of the problem on a fixed domain $\Omega^{\square}$, which introduces a prolongation $\tilde{u}$ of the solution $u$. It yields a weak formulation of the parametric (stochastic) problem in a tensor product space $\mathcal{V}^{\square} \otimes \mathcal{S}$ (product of space functions and parametric functions $)^{2}$, with $\mathcal{V}^{\square} \subset\left\{w: \Omega^{\square} \rightarrow \mathbb{R}\right\}$ and $\mathcal{S} \subset\{\lambda: \Xi \rightarrow \mathbb{R}\}$.

Model reduction techniques based on the construction of separated representations are receiving a growing interest in scientific computing. A family of methods, recently called Proper Generalized Decomposition (PGD) methods have been introduced for the a priori construction of separated representations of the solution of problems defined in tensor product spaces $[1,12,20,6,8]$. PGD methods can be interpreted as generalizations of Proper Orthogonal Decomposition (or Singular Value Decomposition, or Karhunen-Loeve Decomposition) for the a priori construction of a separated representation of the solution. In the context of uncertainty propagation, this method has been initially introduced as a generalization of spectral decompositions [17] for the a priori construction of a separated representation of the solution of stochastic PDEs.

\footnotetext{
$\overline{2}$ In the model example, $\mathcal{V}^{\square} \subset H^{1}\left(\Omega^{\square}\right)$ and $\mathcal{S}=L_{P_{\xi}}^{2}(\Xi)$ with $P_{\xi}$ a measure associated with $\xi$.
} 
The solution is here searched under the form

$$
u(x, \xi) \approx \sum_{i=1}^{m}\left(w_{i} \otimes \lambda_{i}\right)(x, \xi)=\sum_{i=1}^{m} w_{i}(x) \lambda_{i}(\xi)
$$

with $w_{i} \in \mathcal{V}^{\square}$ and $\lambda_{i} \in \mathcal{S}$. Several definitions of separated representations have been proposed. In this paper, we introduce a particular progressive definition of the PGD, based on successive best approximation problems for the progressive definition of the couples of functions $\left(w_{i}, \lambda_{i}\right) \in \mathcal{V}^{\square} \times \mathcal{S}$, which are constructed with an alternated minimization procedure ${ }^{3}$. This construction only requires the solution of independent subproblems defined on $\mathcal{S}$ (parametric algebraic equations) and subproblems defined on $\mathcal{V}^{\square}$ (non parametric PDEs). Let us note that PGD methods have also been extended to uncertainty quantification problems for high-dimensional parametric models [7,21] (i.e. involving a large number of parameters $\left.\xi=\left(\xi_{1}, \ldots, \xi_{r}\right) \in \Xi \subset \mathbb{R}^{r}\right)$ by using separated variables representations of the solution $u\left(x, \xi_{1}, \ldots, \xi_{r}\right) \approx \sum_{i=1}^{m} w_{i}(x) \lambda_{i}^{1}\left(\xi_{1}\right) \ldots \lambda_{i}^{r}\left(\xi_{r}\right)$. These methods allow the a priori construction of a separated representation of a solution defined on a very high-dimensional parametric space. The method can handle problems with such a dimension that their solution is unfeasible with classical spectral stochastic approximation techniques. For the sake of simplicity, in this article, we restrict the presentation to the construction of a separation of type (1), without any additional separation of parameters $\xi=\left(\xi_{1}, \ldots, \xi_{r}\right)$. However, following [21], the methodology could be easily extended to the case of multidimensional separations.

The outline of the paper is as follows. In section 2, we introduce a fictitious domain formulation of partial differential equations defined on uncertain parameterized domains, resulting in a weak formulation defined in a tensor product space, where variational forms involve the indicator function of the parameterized domain. In section 3, we introduce and illustrate the Proper Generalized Decomposition (PGD) method for the construction of a separated representation (1) of the solution. In order to obtain an efficient method outperforming traditional solution techniques, variational forms must also admit accurate low rank separated representations. For this purpose, in section 4, we introduce separated representations of the indicator function in order to obtain accurate separated representations of variational forms. Smoothing of indicator functions is introduced in order to improve the convergence rate of their separated representations. Moreover, a method is proposed for the construction of a constrained tensor product approximation which preserves positivity and therefore ensures well-posedness of problems associated with approximate indicator functions. In section 5, a second example illustrates the overall methodology.

\footnotetext{
$\overline{3}$ Alternative definitions and improved algorithms for their construction could be also used $[18,20]$.
} 


\section{Formulation of the problem and fictitious domain approach}

\subsection{Partial differential equation defined on uncertain parameterized domain}

Let $\left(\Xi, \mathcal{B}, P_{\xi}\right)$ be a finite dimensional probability space representing the uncertainties on a geometry ${ }^{4}$, where $\Xi \subset \mathbb{R}^{r}$ is the set of elementary events ${ }^{5} . P_{\xi}$ denotes the probability measure of a finite set of random variables $\xi$ which are random parameters controlling the shape of a random domain $\Omega(\xi) \subset \mathbb{R}^{d}{ }^{6}$ For each $\xi \in \Xi$, the domain $\Omega(\xi)$ is bounded and connected and its boundary $\partial \Omega(\xi)$ is assumed to be sufficiently smooth (e.g. curvilinear polygonal or piecewise $C^{1,1}$ boundary). We here consider a simple diffusion partial differential equation defined on random domain $\Omega(\xi)$

$$
\begin{aligned}
& -\Delta u=f \quad \text { on } \quad \Omega(\xi) \\
& \nabla u \cdot n=0 \text { on } \Gamma_{N}(\xi) \\
& u=0 \text { on } \Gamma_{D}(\xi)
\end{aligned}
$$

where $\Gamma_{D}$ and $\Gamma_{N}$ are homogeneous Dirichlet and Neumann boundaries respectively, with $\Gamma_{D} \cap \Gamma_{N}=\emptyset$ and $\Gamma_{D} \cup \Gamma_{N}=\partial \Omega$. The spatial-weak and stochastic-strong formulation of (2) writes $^{7}$ :

$$
\begin{aligned}
& \text { Find } u(\xi) \in \mathcal{V}(\xi) \text { such that } \\
& a(u(\xi), v ; \xi)=\ell(v ; \xi) \quad \forall v \in \mathcal{V}(\xi)
\end{aligned}
$$

with

$$
a(u, v ; \xi)=\int_{\Omega(\xi)} \nabla v \cdot \nabla u d x, \quad \ell(v ; \xi)=\int_{\Omega(\xi)} v f d x
$$

and where

$$
\mathcal{V}(\xi)=\left\{v \in H^{1}(\Omega(\xi)) ; v=0 \text { on } \Gamma_{D}(\xi)\right\}
$$

$\overline{4}$ In this article, we consider that geometrical uncertainties are modeled within a probabilistic framework. However, let us note that for classical parametric analyses, the set $\Xi$ could be endowed with a uniform (non probability) measure $P_{\xi}$.

5 Note that random domains with boundaries defined by infinite dimensional random fields could be handled by introducing a first stochastic discretization step (e.g. with Karhunen-Loeve decomposition).

6 More precisely, $\Omega$ is considered as a random variable defined on $\left(\Xi, \mathcal{B}, P_{\xi}\right)$ with values in the set of measurable sets in $\mathbb{R}^{d}$.

7 We consider equivalently $u$ as a function of $\xi$ with values in $H^{1}(\Omega(\xi))$, denoted $u(\xi)(x)$, or a function of $x$ and $\xi$ with values in $\mathbb{R}$, denoted $u(x, \xi)$. 
We now introduce the function space

$$
\mathcal{W}=\left\{v: y \in \Xi \mapsto v(y) \in \mathcal{V}(y) ; \int_{\Xi}\|v(y)\|_{\mathcal{V}(y)}^{2} d P_{\xi}(y)<\infty\right\}:=L_{P_{\xi}}^{2}(\Xi ; \mathcal{V})
$$

with

$$
\|v(y)\|_{\mathcal{V}(y)}^{2}=\int_{\Omega(y)} \nabla v(x) \cdot \nabla v(x) d x .
$$

We denote by $\|\cdot\|_{\mathcal{W}}$ the norm on $\mathcal{W}$ defined by

$$
\|v\|_{\mathcal{W}}^{2}=\int_{\Xi}\|v(y)\|_{\mathcal{V}(y)}^{2} d P_{\xi}(y)
$$

A stochastic-weak formulation can then be written

$$
\begin{aligned}
& \text { Find } u \in \mathcal{W} \text { such that } \\
& A(u, v)=L(v) \quad \forall v \in \mathcal{W}
\end{aligned}
$$

where

$$
\begin{aligned}
& A(u, v)=\int_{\Xi} a(u, v ; y) d P_{\xi}(y)=\int_{\Xi} \int_{\Omega(y)} \nabla u \cdot \nabla v d x d P_{\xi}(y) \\
& L(v)=\int_{\Xi} \ell(v ; y) d P_{\xi}(y)=\int_{\Xi} \int_{\Omega(y)} f v d x d P_{\xi}(y)
\end{aligned}
$$

In this paper, we only consider the case where the Dirichlet boundary $\Gamma_{D}$ is deterministic. The case where $\Gamma_{D}(\xi)$ is random will be briefly discussed in section 2.2.4 and a dedicated method will be presented in another paper. In this paper, we focus on the modelling of geometrical uncertainties. However, note that other sources of uncertainties, on the source term $f$ or on the diffusion operator, can be handled with no extra difficulty.

\subsection{A fictitious domain formulation}

We now introduce a reformulation of problem $(\mathrm{P})$ on a deterministic fictitious domain. For simplicity, the present paper is restricted to a simple diffusion equation with homogeneous boundary conditions. However, the results could be naturally extended to a larger class of problems, by using ad-hoc fictitious domain reformulations (see e.g. $[11,25,26]$ for a natural extension to diffusionreaction equations and the introduction of non-homogeneous Dirichlet, Neumann or Robin boundary conditions).

\subsubsection{Introduction of a fictitious domain}

We recall that we only consider the case where $\Gamma_{D}$ is deterministic and $\Gamma_{N}(\xi)$ is random. We introduce a deterministic fictitious domain $\Omega^{\square}$ such that $\bigcup_{y \in \Xi} \Omega(y) \subset$ 
$\Omega^{\square}$ and such that $\Gamma_{D} \subset \Gamma_{D}^{\square} \subset \partial \Omega^{\square}$, where the boundary of the fictitious domain $\partial \Omega^{\square}$ is partitioned into two deterministic disjoint sets $\Gamma_{N}^{\square}$ and $\Gamma_{D}^{\square}$ (see Figure 1) ${ }^{8}$.

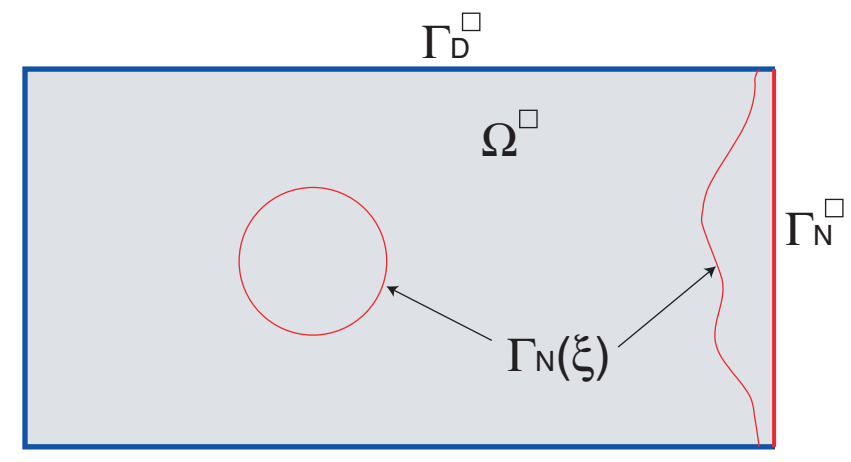

Figure 1. Fictitious domain

\subsubsection{Indicator functions}

We introduce a function $I^{\eta}: \Omega^{\square} \times \Xi \rightarrow \mathbb{R}$, with $0 \leq \eta \leq 1$, defined by

$$
I^{\eta}(x, y)= \begin{cases}1 & \text { if } x \in \Omega(y) \\ \eta & \text { if } x \notin \Omega(y)\end{cases}
$$

$I^{0}(\cdot, y)$ corresponds to the classical indicator function of domain $\Omega(y)$, simply denoted $I$ in the following. Function $I^{\eta}$ can be expressed in terms of $I$ as follows:

$$
I^{\eta}=I+\eta(1-I)=\eta+(1-\eta) I
$$

In practice, we introduce a function $\phi: \Omega^{\square} \times \Xi \rightarrow \mathbb{R}$ such that

$$
I(x, y)=H(-\phi(x, y))
$$

where $H$ is the heaviside function defined by $H(a)=\left\{\begin{array}{ll}1 & \text { if } a>0 \\ 0 & \text { if } a \leq 0\end{array}\right.$. Here, we use for $\phi(x, \xi)$ a random level-set function whose iso-zero in $\Omega^{\square}$ characterizes the random boundary $\Gamma_{N}(\xi)$.

\footnotetext{
8 Note that the choice of boundary conditions on $\partial \Omega^{\square} \backslash \partial \Omega(\xi)$ has a part of arbitrariness. However, this choice does not significantly impact on the solution if the fictitious boundary is chosen sufficiently large.
} 


\subsubsection{Reformulation of the problem}

Problem (3) is reformulated as follows:

$$
\begin{aligned}
& \text { Find } u^{\eta}(\xi) \in \mathcal{V}^{\square} \text { such that } \\
& a^{\eta}\left(u^{\eta}(\xi), v ; \xi\right)=\ell(v ; \xi) \quad \forall v \in \mathcal{V}^{\square}
\end{aligned}
$$

where

$$
\begin{aligned}
& a^{\eta}(u, v ; \xi)=\int_{\Omega^{\square}} I^{\eta} \nabla v \cdot \nabla u d x, \\
& \ell(v ; \xi)=\int_{\Omega^{\square}} I v f d x
\end{aligned}
$$

and where $\mathcal{V}^{\square}$ is a deterministic function space defined by

$$
\mathcal{V}^{\square}=\left\{v \in H^{1}\left(\Omega^{\square}\right) ; v=0 \text { on } \Gamma_{D}^{\square}\right\}
$$

The deterministic counterpart of the above formulation belongs to the class of reformulations proposed in [11]. We now introduce the function space

$$
\mathcal{W}^{\square}=\left\{v: y \in \Xi \mapsto v(y) \in \mathcal{V}^{\square} ; \int_{\Xi}\|v(y)\|_{\mathcal{V} \square}^{2} d P_{\xi}(y)<\infty\right\}=L_{P_{\xi}}^{2}\left(\Xi ; \mathcal{V}^{\square}\right)
$$

with

$$
\|v\|_{\mathcal{V} \square}^{2}=\int_{\Omega^{\square}} \nabla v(x) \cdot \nabla v(x) d x .
$$

Since $\mathcal{V}^{\square}$ is deterministic, we have $\mathcal{W}^{\square}=L_{P_{\xi}}^{2}\left(\Xi ; \mathcal{V}^{\square}\right) \simeq \mathcal{V}^{\square} \otimes \mathcal{S}$, where $\mathcal{S}:=$ $L_{P_{\xi}}^{2}(\Xi) . \mathcal{W}^{\square}$ is equipped with the following norm

$$
\|v\|_{\mathcal{W} \square}^{2}=\int_{\Xi}\|v(y)\|_{\mathcal{V} \square}^{2} d P_{\xi}(y)=\int_{\Xi} \int_{\Omega^{\square}}|\nabla v(x, y)|^{2} d \mu(x, y)
$$

where $\mu$ is the product measure on $\mathbb{R}^{d} \times \Xi$, such that $d \mu(x, y)=d x d P_{\xi}(y)$. We then have the following stochastic-weak formulation of problem (10):

$$
\begin{aligned}
& \text { Find } u^{\eta} \in \mathcal{W}^{\square}=\mathcal{V}^{\square} \otimes \mathcal{S} \text { such that } \\
& A^{\eta}\left(u^{\eta}, v\right)=L(v) \quad \forall v \in \mathcal{W}^{\square}=\mathcal{V}^{\square} \otimes \mathcal{S}
\end{aligned}
$$

where $A^{\eta}$ and $L$ are continuous bilinear and linear forms on $\mathcal{W}^{\square}=\mathcal{V}^{\square} \otimes \mathcal{S}$, defined by

$$
\begin{aligned}
& A^{\eta}(u, v)=\int_{\Xi} a^{\eta}(u, v ; y) d P_{\xi}(y)=\int_{\Omega^{\square} \times \Xi} I^{\eta} \nabla v \cdot \nabla u d \mu \\
& L(v)=\int_{\Xi} \ell(v ; y) d P_{\xi}(y)=\int_{\Omega^{\square} \times \Xi} I v f d \mu
\end{aligned}
$$

Using (8), bilinear form $A^{\eta}$ is equivalently expressed in terms of the classical indicator function $I$ as follows:

$$
A^{\eta}(u, v)=\eta \int_{\Omega^{\square} \times \Xi} \nabla v \cdot \nabla u d \mu+(1-\eta) \int_{\Omega^{\square} \times \Xi} I \nabla v \cdot \nabla u d \mu
$$


For $\eta>0, A^{\eta}$ is a symmetric continuous and coercive bilinear form on $\mathcal{W}^{\square}=$ $\mathcal{V}^{\square} \otimes \mathcal{S}$. Therefore, there exists a unique solution to problem $\left(\mathrm{P}^{\eta}\right)$ which is equivalently characterized by

$$
u^{\eta}=\arg \min _{v \in \mathcal{V} \square \otimes \mathcal{S}} \frac{1}{2} A^{\eta}(v, v)-L(v)
$$

The convergence of $u^{\eta}$ when $\eta \rightarrow 0$ is analyzed in appendix A. In the following, we consider that $u^{\eta}$ solution of $\left(\mathrm{P}^{\eta}\right)$ is our reference solution, with $\eta$ chosen sufficiently small such that $u^{\eta}$ provides a good approximation of the solution $u$ of problem $(\mathrm{P})$.

\subsubsection{The case of random Dirichlet boundaries}

The case where Dirichlet boundary $\Gamma_{D}(\xi)$ is random and $\Gamma_{N}$ is deterministic can be handled in the same framework. It corresponds to a penalty method for treating Dirichlet boundary conditions. Let us briefly detail this approach. Let us introduce the partition $\partial \Omega^{\square}=\Gamma_{D}^{\square} \cup \Gamma_{N}^{\square}$ with $\Gamma_{N} \subset \Gamma_{N}^{\square}$. Then, we can use the reformulation $\left(\mathrm{P}^{\eta}\right)$, with the following definition of bilinear form:

$$
A^{\eta}(u, v)=\int_{\Omega^{\square} \times \Xi} I^{\eta} \nabla v \cdot \nabla u d \mu+\int_{\Omega^{\square \times \Xi}} \eta(1-I) v u d \mu
$$

with $I^{\eta}=I$ for a $L^{2}$ penalization or $I^{\eta}=I+\eta(1-I)$ for a $H^{1}$ penalization. We choose $\eta>1$. Denoting $u$ and $u^{\eta}$ the solutions of $(\mathrm{P})$ and $\left(\mathrm{P}^{\eta}\right)$ respectively, we have $\lim _{\eta \rightarrow \infty}\left\|u-u^{\eta}\right\|_{\mathcal{W}}=0$. The treatment of Dirichlet boundary conditions on a random boundary is then possible with a standard Galerkin approximation method, consisting in introducing approximation spaces in formulation $\left(\mathrm{P}^{\eta}\right)$. However, this reformulation is not adapted to the use of Proper Generalized Decomposition, in the case where the indicator function $I$ is approximated (see section 4). Indeed, a coarse approximation of $I$ can have dramatic consequences on the quality of the solution, because $\eta$ takes high values (required in order to correctly treat boundary conditions). An alternative reformulation, adapted to the Proper Generalized Decomposition method, will be introduced in another paper. It consists in using a characteristic function method [3].

\section{Proper Generalized Decomposition}

We consider the solution of problem $\left(\mathrm{P}^{\eta}\right)$ with the Proper Generalized Decomposition (PGD) method. The idea is to find an approximation of the solution 
$u^{\eta} \in \mathcal{V}^{\square} \otimes \mathcal{S}$ of $\left(\mathrm{P}^{\eta}\right)$ under the form ${ }^{9}$

$$
u^{\eta} \approx u_{m}=\sum_{i=1}^{m} w_{i} \lambda_{i}, \quad w_{i} \in \mathcal{V}^{\square}, \quad \lambda_{i} \in \mathcal{S}
$$

where $u_{m}$ is called a rank- $m$ separated representation of $u^{\eta}$. This can be interpreted as a simultaneous construction of reduced bases of spatial functions $\left\{w_{i}\right\}_{i=1}^{m}$ and stochastic functions $\left\{\lambda_{i}\right\}_{i=1}^{m}$, which are optimal (in some sense to be defined) for the representation of the present solution $u^{\eta}$. We here present a particular progressive definition of the PGD. The reader can refer to $[17,18,19,20]$ for alternative definitions. Note that the constructed sequence $u_{m}$ depends on the parameter $\eta$ but this dependence will be omitted in order to simplify the notations.

\subsection{Definition of the progressive PGD}

Definition 1 The progressive $P G D\left\{u_{m}\right\}_{m \in \mathbb{N}}$ of the solution $u^{\eta}$ of problem $\left(\mathrm{P}^{\eta}\right)$ is defined by

$$
u_{m}=u_{m-1}+w_{m} \lambda_{m}
$$

with $u_{0}=0$ and

$$
\left(w_{m}, \lambda_{m}\right) \in \arg \min _{w \in \mathcal{V}^{\square}, \lambda \in \mathcal{S}} \frac{1}{2} A^{\eta}(w \lambda, w \lambda)-L(w \lambda)+A^{\eta}\left(u_{m-1}, w \lambda\right)
$$

Definition 1 is equivalent to ${ }^{10}$

$$
\left(w_{m}, \lambda_{m}\right) \in \arg \min _{w \in \mathcal{V}^{\square}, \lambda \in \mathcal{S}}\left\|u^{\eta}-u_{m-1}-w \lambda\right\|_{A^{\eta}}^{2}
$$

where $u^{\eta}$ is the solution of $\left(\mathrm{P}^{\eta}\right)$ and $\|\cdot\|_{A^{\eta}}$ is the norm on $\mathcal{V}^{\square} \otimes \mathcal{S}$ induced by $A^{\eta}$, defined by

$$
\|v\|_{A^{\eta}}^{2}=A^{\eta}(v, v) .
$$

A new couple of functions $\left(w_{m}, \lambda_{m}\right) \in \mathcal{V}^{\square} \times \mathcal{S}$ is then defined as a couple which minimizes the distance between $u_{m}$ and $u^{\eta}$, with respect to the norm induced by $A^{\eta}$.

Let us introduce the mapping $F_{m}: \mathcal{S} \rightarrow \mathcal{V}^{\square}$ defined by

$$
F_{m}(\lambda)=\arg \min _{w \in \mathcal{V} \square} \frac{1}{2} A^{\eta}(w \lambda, w \lambda)-L(w \lambda)+A^{\eta}\left(u_{m-1}, w \lambda\right)
$$

$\overline{9}$ The tensor product $\otimes$ is omitted in the notation $w \lambda \simeq w \otimes \lambda$.

${ }^{10}$ Note that there exist infinitely many solutions to the minimization problem (19). If a normalization of $w_{m}$ (or $\lambda_{m}$ ) is added, there may still exists different minimizers $\left(w_{m}, \lambda_{m}\right)$. 
and the mapping $F_{m}^{\diamond}: \mathcal{V}^{\square} \rightarrow \mathcal{S}$ defined by

$$
F_{m}^{\diamond}(w)=\arg \min _{\lambda \in \mathcal{S}} \frac{1}{2} A^{\eta}(w \lambda, w \lambda)-L(w \lambda)+A^{\eta}\left(u_{m-1}, w \lambda\right) .
$$

We have the following properties:

$$
\left\|u^{\eta}-u_{m-1}-w F_{m}^{\diamond}(w)\right\|_{A^{\eta}}^{2}=\left\|u^{\eta}-u_{m-1}\right\|_{A^{\eta}}^{2}-r_{m}^{\diamond}(w)
$$

with $r_{m}^{\diamond}(w)=\left\|w F_{m}^{\diamond}(w)\right\|_{A^{\eta}}^{2}$, and

$$
\left\|u^{\eta}-u_{m-1}-F_{m}(\lambda) \lambda\right\|_{A^{\eta}}^{2}=\left\|u^{\eta}-u_{m-1}\right\|_{A^{\eta}}^{2}-r_{m}(\lambda)
$$

with $r_{m}(\lambda)=\left\|F_{m}(\lambda) \lambda\right\|_{A^{\eta}}^{2}$. We then have the following theorem, which is a particular case of a more general convergence result of progressive PGD in tensor product Hilbert spaces [8]. ${ }^{11}$

Theorem 1 The progressive $P G D\left\{u_{m}\right\}_{m \in \mathbb{N}}$ strongly converges with $m$ towards the solution $u^{\eta}$ of problem $\left(\mathrm{P}^{\eta}\right)$ (for a fixed $\eta$ ). We have

$$
\left\|u^{\eta}-u_{m}\right\|_{A^{\eta}}^{2}=\left\|u^{\eta}\right\|_{A^{\eta}}^{2}-\sum_{i=1}^{m} \sigma_{i}^{2} \underset{m \rightarrow \infty}{\longrightarrow} 0
$$

where $\sigma_{m}$ is defined by

$$
\sigma_{m}^{2}=\max _{w \in \mathcal{V}^{\square}} r_{m}^{\diamond}(w)=\max _{\lambda \in \mathcal{S}} r_{m}(\lambda)
$$

Remark 2 (Approximation) In practice, we introduce the approximation spaces $\mathcal{V}_{N}^{\square} \subset \mathcal{V}^{\square}$ and $\mathcal{S}_{P} \subset \mathcal{S}$ and we construct a $P G D$ sequence $\left\{u_{m}\right\}_{m \in \mathbb{N}} \subset$ $\mathcal{V}_{N}^{\square} \otimes \mathcal{S}_{P}$ which converges to the Galerkin approximation $u_{N, P}^{\eta}$ in $\mathcal{V}_{N}^{\square} \otimes \mathcal{S}_{P}$ of the solution $u^{\eta}$ of problem $\left(\mathrm{P}^{\eta}\right)$.

The progressive PGD can be interpreted in terms of successive pseudo eigenproblems on the operators $T_{m}^{\diamond}(w)=r_{m}^{\diamond}(w) F_{m} \circ F_{m}^{\diamond}(w) \cdot r_{m}^{\diamond}$ is interpreted as a pseudo Rayleigh quotient, which is maximized by the optimal function $w_{m}$, called the dominant pseudo eigenfunction of $T_{m}^{\diamond}$. Equivalently, it can be interpreted in terms of successive pseudo eigenproblems on operators $T_{m}(\lambda)=r_{m}(\lambda) F_{m}^{\diamond} \circ F_{m}(\lambda) . r_{m}$ is interpreted as a pseudo Rayleigh quotient, which is maximized by the optimal function $\lambda_{m}$, called the dominant pseudo eigenfunction of $T_{m}$. The values $\sigma_{m}$ are then interpreted as singular values of a progressive (generalized) singular value decomposition of $u^{\eta}$, also named generalized spectral decomposition (see [17]). The reader can refer to [8] for a more

\footnotetext{
${ }^{11}$ Let us note that for $\eta=0$, bilinear form $A^{\eta}$ only defines a pseudo norm on the tensor Hilbert spaces. However, Theorem 1 is still valid and convergence of the separated representation can be proved with respect to this pseudo-norm (equivalent to a convergence towards a particular solution of problem $\left(\mathrm{P}^{\eta}\right)$ ).
} 
general interpretation in terms of a generalized singular value decomposition in tensor Hilbert spaces.

\subsection{Associated algorithm}

Starting from an initial function $\lambda^{0} \in \mathcal{S}$, we construct the sequences $\left\{w^{k}\right\}_{k \geq 1} \subset$ $\mathcal{V}^{\square}$ and $\left\{\lambda^{k}\right\}_{k \geq 1} \subset \mathcal{S}$, defined by

$$
w^{k+1}=F_{m}\left(\lambda^{k}\right), \quad \lambda^{k+1}=F_{m}^{\diamond}\left(w^{k+1}\right)
$$

The algorithm can be interpreted as an alternated minimization algorithm for solving optimization problem (18). It can also be interpreted as a power-type algorithm ${ }^{12}$ for finding the dominant pseudo eigenfunction of operator $T_{m}^{\diamond}$, or equivalently of $T_{m}$ (see [18]). In practice, only a few iterations (3 or 4) are sufficient to obtain a good approximation of an optimal couple $\left(w_{m}, \lambda_{m}\right)$.

\subsubsection{Application of mapping $F_{m}$ (spatial problem)}

For a given $\lambda \in \mathcal{S}, w=F_{m}(\lambda)$ is the solution of the following problem

$$
w \in \mathcal{V}^{\square}, \quad a_{\lambda}\left(w, w^{*}\right)=l_{\lambda}\left(w^{*}\right) \quad \forall w^{*} \in \mathcal{V}^{\square}
$$

with

$$
\begin{aligned}
& a_{\lambda}\left(w, w^{*}\right)=\int_{\Omega^{\square}} \kappa \nabla w \cdot \nabla w^{*} d x, \\
& l_{\lambda}\left(w^{*}\right)=\int_{\Omega^{\square}} g w^{*} d x-\sum_{i=1}^{m-1} \int_{\Omega^{\square}} \kappa_{i} \nabla w_{i} \cdot \nabla w^{*} d x,
\end{aligned}
$$

and

$$
\begin{aligned}
& \kappa(x)=\int_{\Xi} I^{\eta}(x, y) \lambda(y)^{2} d P_{\xi}(y)=\mathbb{E}\left(I^{\eta}(x, \xi) \lambda(\xi)^{2}\right) \\
& g(x)=\int_{\Xi} I(x, y) f(x, y) \lambda(y) d P_{\xi}(y)=\mathbb{E}(I(x, \xi) f(x, \xi) \lambda(\xi)) \\
& \kappa_{i}(x)=\int_{\Xi} I^{\eta}(x, y) \lambda(y) \lambda_{i}(y) d P_{\xi}(y)=\mathbb{E}\left(I^{\eta}(x, \xi) \lambda(\xi) \lambda_{i}(\xi)\right)
\end{aligned}
$$

where $\mathbb{E}$ is the mathematical expectation defined by $\mathbb{E}(f(\xi))=\int_{\Xi} f(y) d P_{\xi}(y)$.

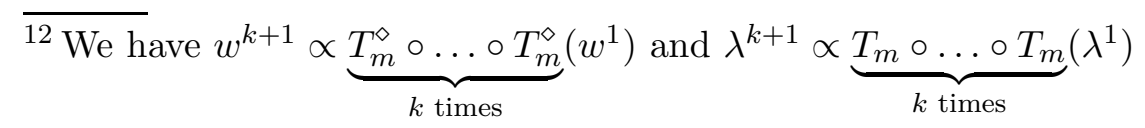


3.2.1.1 Interpretation Problem $\left(\mathrm{P}_{w}\right)$ appears as a classical diffusion equation on $\Omega^{\square}$ the strong formulation of which reads:

$$
\begin{aligned}
& -\nabla \cdot(\kappa \nabla w)=g+\sum_{i=1}^{m-1} \nabla \cdot\left(\kappa_{i} \nabla w_{i}\right) \quad \text { on } \quad \Omega^{\square} \\
& \kappa \nabla w \cdot n=-\sum_{i=1}^{m-1} \kappa_{i} \nabla w_{i} \cdot n \quad \text { on } \quad \Gamma_{N}^{\square} \\
& w=0 \quad \text { on } \quad \Gamma_{D}^{\square}
\end{aligned}
$$

$\kappa$ appears as a non homogeneous diffusion coefficient of a virtual material. Let us consider that $\lambda$ is normalized, i.e. $\|\lambda\|^{2}=\mathbb{E}\left(\lambda^{2}\right)=1$. Denoting $\Xi_{x}=\{y \in$ $\Xi ; x \in \Omega(y)\}$, we have

$$
\begin{aligned}
\kappa(x) & =\int_{\Xi_{x}} \lambda^{2} d P_{\xi}+\eta \int_{\Xi \backslash \Xi_{x}} \lambda^{2} d P_{\xi} \\
& =1+(\eta-1) \int_{\Xi \backslash \Xi_{x}} \lambda^{2} d P_{\xi}=\eta+(1-\eta) \int_{\Xi_{x}} \lambda^{2} d P_{\xi}
\end{aligned}
$$

We have $\kappa(x)=1$ (resp. $\eta$ ) for $x$ such that $P_{\xi}\left(\Xi_{x}\right)=1$ (resp. 0), i.e. if point $x$ is almost surely inside (resp. outside) the domain. For $x$ such that $0<P_{\xi}\left(\Xi_{x}\right)<1$, we have $\eta<\kappa(x)<1$ (since $\eta<1$ ).

3.2.1.2 Approximation In practice, we introduce a classical finite element approximation space $\mathcal{V}_{N}^{\square} \subset \mathcal{V}^{\square}$ and an approximation $w_{N}$ of $w$ is defined by:

$$
w_{N} \in \mathcal{V}_{N}^{\square}, \quad a_{\lambda}\left(w_{N}, w^{*}\right)=l_{\lambda}\left(w^{*}\right) \quad \forall w^{*} \in \mathcal{V}_{N}^{\square}
$$

\subsubsection{Application of mapping $F_{m}^{\diamond}$ (stochastic algebraic equation)}

For a given $w \in \mathcal{V}^{\square}, \lambda=F_{m}^{\diamond}(w)$ is the solution of the following problem

$$
\lambda \in \mathcal{S}, \quad a_{w}\left(\lambda, \lambda^{*}\right)=l_{w}\left(\lambda^{*}\right) \quad \forall \lambda^{*} \in \mathcal{S}
$$

with

$$
\begin{aligned}
& a_{w}\left(\lambda, \lambda^{*}\right)=\mathbb{E}\left(\lambda^{*} \alpha \lambda\right), \\
& l_{w}\left(\lambda^{*}\right)=\mathbb{E}\left(\lambda^{*}\left(\beta-\sum_{i=1}^{m-1} \alpha_{i} \lambda_{i}\right)\right),
\end{aligned}
$$


where

$$
\begin{aligned}
& \alpha(y)=\int_{\Omega^{\square}} I^{\eta}(x, y) \nabla w(x) \cdot \nabla w(x) d x \\
& \beta(y)=\int_{\Omega^{\square}} I(x, y) f(x, y) w(x) d x \\
& \alpha_{i}(y)=\int_{\Omega^{\square}} I^{\eta}(x, y) \nabla w(x) \cdot \nabla w_{i}(x) d x
\end{aligned}
$$

3.2.2.1 Approximation In practice, we introduce a classical spectral stochastic approximation space $\mathcal{S}_{P} \subset \mathcal{S}$ and an approximation $\lambda_{P}$ of $\lambda$ is defined by:

$$
\lambda_{P} \in \mathcal{S}_{P}, \quad a_{w}\left(\lambda_{P}, \lambda^{*}\right)=l_{w}\left(\lambda^{*}\right) \quad \forall \lambda^{*} \in \mathcal{S}_{P}
$$

\subsection{Illustration}

\subsubsection{Description of the problem}

We consider the Poisson equation (2) with $f=1$ and a domain $\Omega(\xi)=$ $\{(0,2) \times(0,1)\} \backslash D(\xi)$, where $D(\xi)=\left\{x \in \mathbb{R}^{2} ;|x-c(\xi)| \leq R\right\}$ is a random circular domain with radius $R=0.2$ and center $c(\xi)=(1.4 \xi+0.3,0.5)$, where $\xi$ is a uniform random variable on $\Xi=(0,1)$ (see Figure 2 ). We consider the fictitious domain $\Omega^{\square}=(0,2) \times(0,1)$ such that $\Omega(\xi)=\Omega^{\square} \backslash D(\xi)$. We consider homogeneous Dirichlet boundary conditions on $\Gamma_{D}=\partial \Omega^{\square}$ and homogeneous Neumann boundary conditions on $\Gamma_{N}(\xi)=\partial D(\xi)$. We have $\Gamma_{D}^{\square}=\partial \Omega^{\square}$ and $\Gamma_{N}^{\square}=\emptyset$. The circle is characterized by the iso-zero in $\Omega^{\square}$ of the parameterized level-set function $\phi(x, \xi)=R-|x-c(\xi)|$.

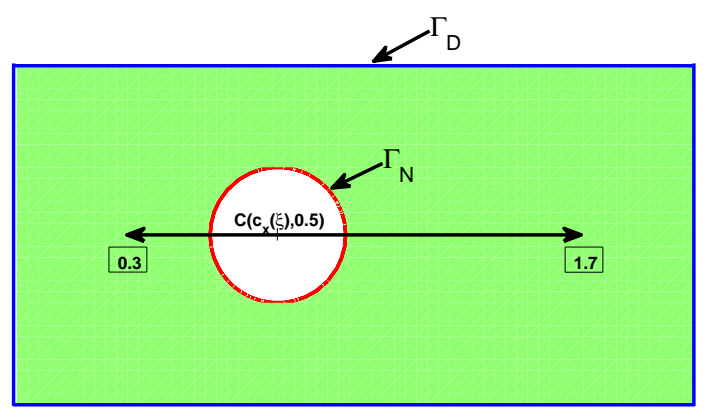

Figure 2. Model example.

A finite element approximation space $\mathcal{V}_{N}^{\square}$ is introduced using a regular mesh composed of 3660 triangular elements $(N=1740)$. We also introduce an approximation space $\mathcal{S}_{P}$ composed of piecewise polynomial approximation of degree 2 (of finite element type) associated with a uniform partition of $\Xi$ into 20 elements $(P=60)$. In the following, the Galerkin approximation $u_{N, P}^{\eta} \in$ $\mathcal{V}_{N}^{\square} \otimes \mathcal{S}_{P}$ of problem $\left(\mathrm{P}^{\eta}\right)$ will be simply denoted by $u^{\eta}$. 


\subsubsection{Behavior of the fictitious domain method}

A convergence analysis of the fictitious method with respect to $\eta$ is first conducted. The solution $u^{\eta}$ of $\left(\mathrm{P}^{\eta}\right)$ is compared to the solution $u$ of the initial problem $(\mathrm{P})$ by introducing the error indicator

$$
\varepsilon_{\Omega}\left(u^{\eta} ; u\right)=\frac{\left\|u-u^{\eta}\right\|_{\Omega}}{\|u\|_{\Omega}}
$$

with $\|\cdot\|_{\Omega}$ the natural norm on $L_{P_{\xi}}^{2}\left(\Xi ; L^{2}(\Omega)\right)$ defined by

$$
\|u\|_{\Omega}^{2}=\int_{\Xi} \int_{\Omega(y)} u^{2} d x d P_{\xi}(y)
$$

Solution $u$ is obtained with the same spatial mesh as for $u^{\eta}$. The convergence of $u^{\eta}$ is illustrated in Figure 3. We observe a linear convergence of $u^{\eta}$ with $\eta$. This illustrates the convergence result (A.2) stated in appendix A.

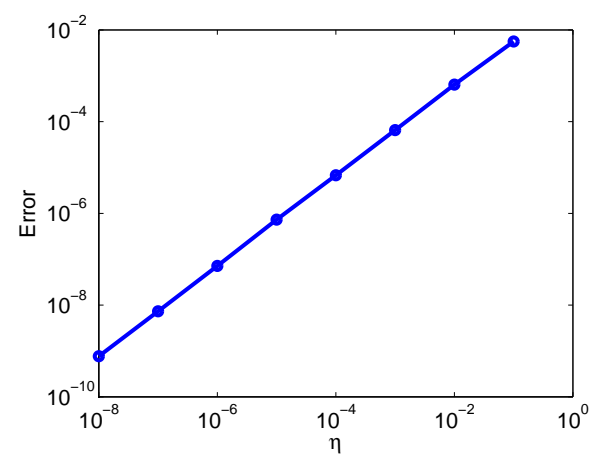

Figure 3. Convergence of the solution of the fictitious domain method: evolution of the error indicator $\varepsilon_{\Omega}\left(u^{\eta} ; u\right)$ with $\eta$.

In order to study the transmission condition on the internal boundary $\Gamma_{N}$ recalled in remark 4 of appendix A, we introduce the following indicator:

$$
i_{B C}(\eta)=\frac{\left\|\frac{1}{\operatorname{meas}\left(\Gamma_{N}\right)^{1 / 2}} \frac{\partial u^{\eta}}{\partial n}\right\|_{\Gamma_{N}}}{\left\|\frac{1}{\text { meas }(\Omega)^{1 / 2}} \nabla u^{\eta}\right\|_{\Omega}}
$$

where $n$ is the unit normal to $\Gamma_{N} \cdot i_{B C}(\eta)$ is evaluated for different spatial meshes and is plotted in Figure 4.

We observe that as $\eta$ tends towards 0 , the homogeneous Neumann boundary condition is better satisfied until the plateau due to the finite element approximation is reached. 


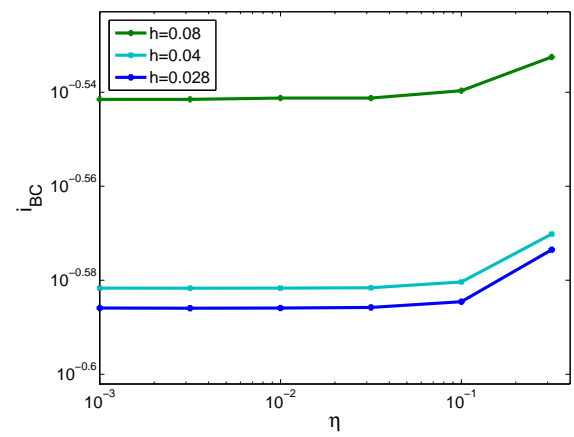

Figure 4. Evaluation of the transmission condition on $\Gamma_{N}$ : evolution of indicator $i_{B C}(\eta)$ with $\eta$ for different element sizes $h$ of the spatial mesh.

\subsubsection{Convergence of the PGD}

We now adopt a fictitious domain formulation with $\eta=0.01$ and we consider as a reference solution the Galerkin approximation $u^{\eta} \in \mathcal{V}_{N}^{\square} \otimes \mathcal{S}_{P}$ of problem $\left(\mathrm{P}^{\eta}\right)$.

The progressive PGD $u_{m}$ is compared to the reference solution $u^{\eta} \in \mathcal{V}_{N}^{\square} \otimes \mathcal{S}_{P}$ with the error indicator $\varepsilon_{\Omega}\left(u_{m} ; u^{\eta}\right)$. The convergence of the PGD is illustrated in Figure 5. We observe a fast convergence of the PGD and the error $\varepsilon_{\Omega}\left(u_{m} ; u^{\eta}\right)=10^{-2}$ is reached with a low rank $m=10$. Figures 6 and 7 respec-

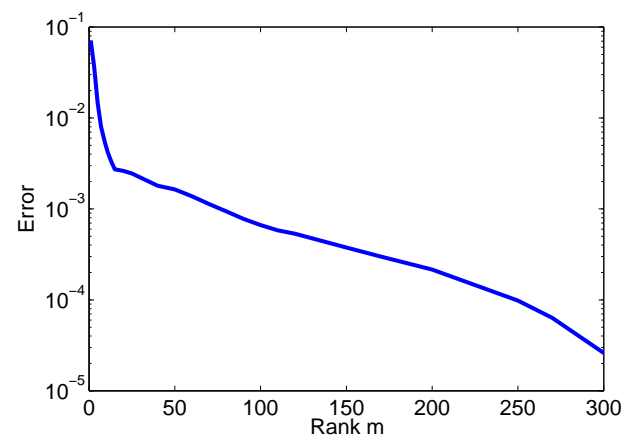

Figure 5. Convergence of the progressive PGD: evolution of the error indicator $\varepsilon_{\Omega}\left(u_{m} ; u^{\eta}\right)$ with $m$.

tively show the first deterministic vectors $w_{i}$ and parametric functions $\lambda_{i}$ of the PGD. We can observe that the first sets of functions are smooth functions that can describe the global behavior of the response. Then as the progressive PGD goes along, the new sets of functions allow to well describe the local behavior of the response. Figure 8 illustrates the first diffusion coefficients $\kappa(x)=\mathbb{E}\left(I^{\eta} \lambda_{i}^{2}\right)$ of the "virtual material" defined in (28). The black areas correspond to $\kappa=1$ and the white areas correspond to $\kappa=\eta$. We verify that $\kappa(x)=1$ for points $x$ almost surely inside the random domain $\Omega(\xi)$. Figure 9 shows the reference solution $u^{\eta}$ and $u_{m}$ for some values of rank $m$, evaluated for $\xi=0.5$. Again, it illustrates the good convergence of the PGD towards the 


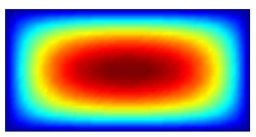

(a) $w_{1}$

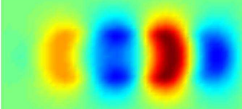

(e) $w_{5}$

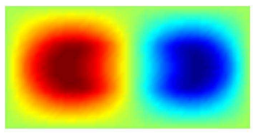

(b) $w_{2}$

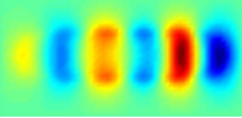

(f) $w_{6}$

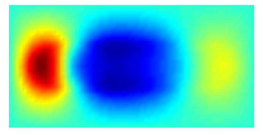

(c) $w_{3}$

\section{(1)}

(g) $w_{7}$

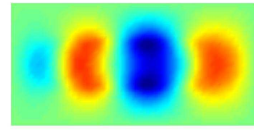

(d) $w_{4}$

Figure 6. The first deterministic vectors $w_{i}$ of the PGD.

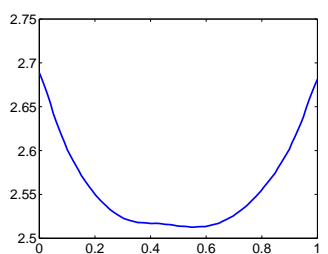

(a) $\lambda_{1}$

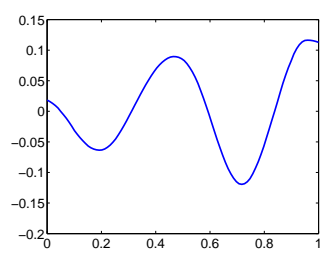

(e) $\lambda_{5}$

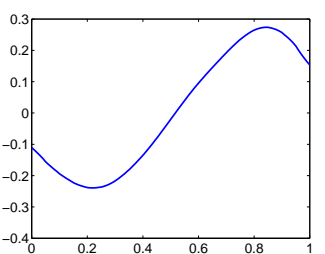

(b) $\lambda_{2}$

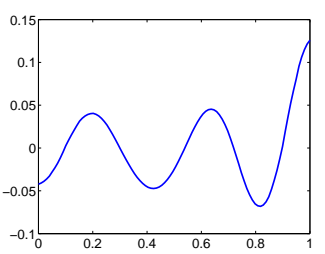

(f) $\lambda_{6}$

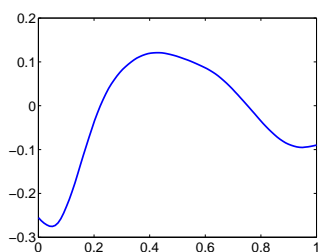

(c) $\lambda_{3}$

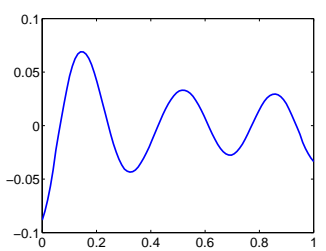

(g) $\lambda_{7}$

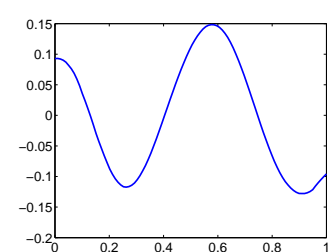

(d) $\lambda_{4}$

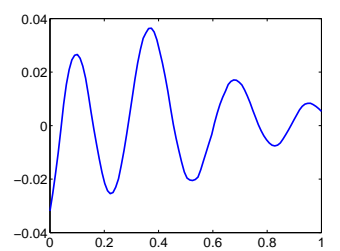

(h) $\lambda_{8}$

Figure 7. The first parametric functions $\lambda_{i}(\xi)$ of the PGD.

reference solution. Finally, Figure 10 shows the reference solution $u^{\eta}$ and the PGD approximation $u_{60}$ for several realizations of $\xi$. The rank-60 progressive PGD gives a very accurate approximation for all these realizations. 

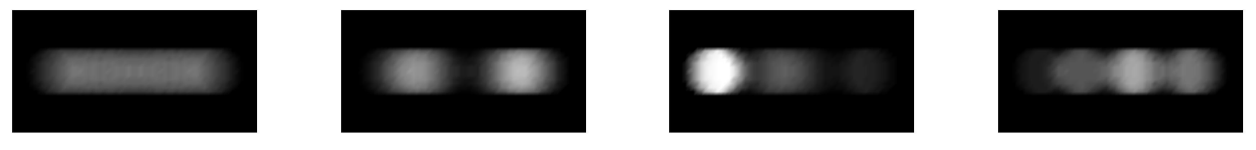
(a) $\mathbb{E}\left(I^{\eta} \lambda_{1}^{2}\right)$
(b) $\mathbb{E}\left(I^{\eta} \lambda_{2}^{2}\right)$
(c) $\mathbb{E}\left(I^{\eta} \lambda_{3}^{2}\right)$
(d) $\mathbb{E}\left(I^{\eta} \lambda_{4}^{2}\right)$
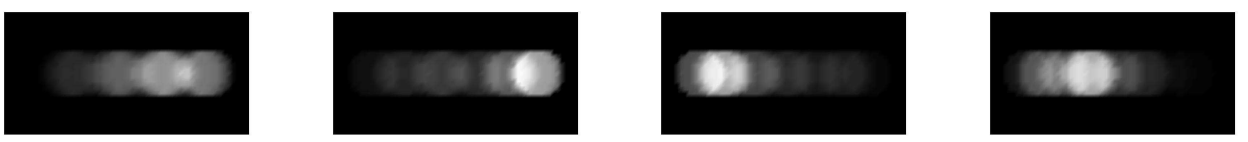
(e) $\mathbb{E}\left(I^{\eta} \lambda_{5}^{2}\right)$
(f) $\mathbb{E}\left(I^{\eta} \lambda_{6}^{2}\right)$
(g) $\mathbb{E}\left(I^{\eta} \lambda_{7}^{2}\right)$
(h) $\mathbb{E}\left(I^{\eta} \lambda_{8}^{2}\right)$

Figure 8. The first diffusion coefficient $\kappa=\mathbb{E}\left(I^{\eta} \lambda_{i}^{2}\right)$ of the "virtual material".

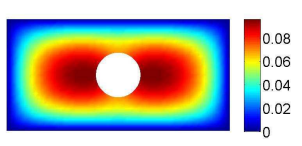

(a) $u^{\eta}$

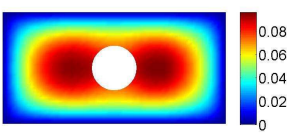

(d) $u_{5}$

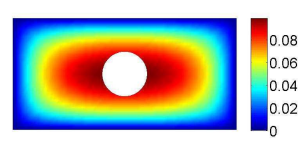

(b) $u_{1}$

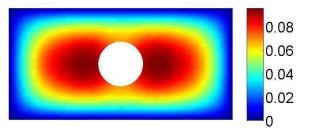

(e) $u_{9}$

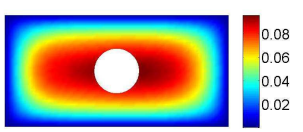

(c) $u_{3}$

Figure 9. Comparison between $u^{\eta}(\cdot, \xi)$ and $u_{m}(\cdot, \xi)$ for $\xi=0.5$.
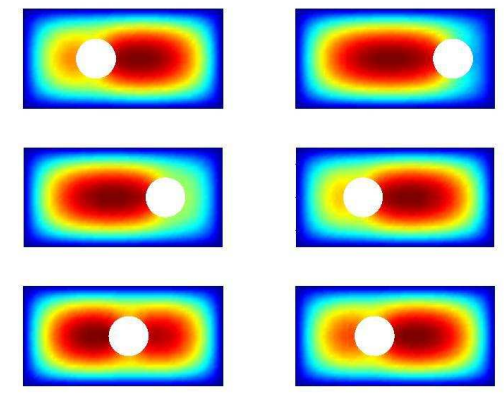

(a)
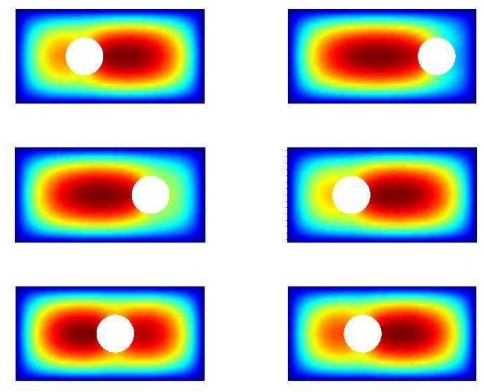

Figure 10. Comparison between $u^{\eta}(\cdot, \xi)$ (a) and $u_{60}(\cdot, \xi)$ (b) for 6 realizations of $\xi$. 


\section{Separated representations of the indicator functions}

When directly applied to problem $\left(\mathrm{P}^{\eta}\right)$, the PGD method is not computationally tractable since bilinear form $A^{\eta}$ and linear form $L$ do not have a "separated form". In practice, when the approximation spaces $\mathcal{V}_{N}^{\square} \subset \mathcal{V}^{\square}$ and $\mathcal{S}_{P} \subset \mathcal{S}$ are introduced, the solutions of problems $\left(\mathrm{P}_{w}^{N}\right)$ and $\left(\mathrm{P}_{\lambda}^{P}\right)$ require a fine integration of the bilinear and linear forms, which limits the use of the method to relatively coarse approximation spaces at both spatial and stochastic levels. This point was also the limiting point of the direct Galerkin approximation approach introduced in [23], where specific integration techniques (computationally costly) were proposed.

We here propose to introduce a suitable approximation of the indicator function $I$ in order to obtain a separated representation of bilinear and linear forms and therefore to obtain a significant gain with the PGD method compared to a classical direct computation of the Galerkin approximation of problem $\left(\mathrm{P}^{\eta}\right)$.

\subsection{Reformulation of the problem with approximate indicator functions}

We introduce the following rank-s separated representation of $I$ :

$$
I(x, \xi) \approx I_{s}(x, \xi)=\sum_{i=1}^{s} g_{i}(x) \chi_{i}(\xi)
$$

and introduce the associated problem:

$$
\begin{aligned}
& \text { Find } u^{\eta, s} \in \mathcal{W}^{\square}=\mathcal{V}^{\square} \otimes \mathcal{S} \text { such that } \\
& A_{s}^{\eta}\left(u^{\eta, s}, v\right)=L_{s}^{\eta}(v) \quad \forall v \in \mathcal{W}^{\square}=\mathcal{V}^{\square} \otimes \mathcal{S}
\end{aligned}
$$

where $A_{s}^{\eta}$ and $L_{s}^{\eta}$ are approximations of the bilinear and linear forms $A^{\eta}$ and $L$, obtained by replacing $I$ by $I_{s}$ in equations (15) and (14):

$$
\begin{aligned}
& A_{s}^{\eta}(u, v)=\eta \int_{\Omega^{\square} \times \Xi} \nabla u \cdot \nabla v d \mu+(1-\eta) \int_{\Omega^{\square} \times \Xi} I_{s} \nabla u \cdot \nabla v d \mu \\
& L_{s}^{\eta}(v)=\int_{\Omega^{\square} \times \Xi} I_{s} f^{\eta} v d \mu
\end{aligned}
$$

where $f^{\eta}$ is a prolongation of $f$ on $\Omega^{\square} \times \Xi$ which can possibly depend on parameter $\eta$.

Two important issues must be considered: the separated representation (38) should be such that (i) problem $\left(\mathrm{P}^{\eta, s}\right)$ is well posed and (ii) $u^{\eta, s}$ is a "good approximation" of the solution $u^{\eta}$ of problem $\left(\mathrm{P}^{\eta}\right)$. These two issues are solved 
if there exists an integer $s^{\prime}$ such that for all $s \geq s^{\prime}$, we have (almost surely)

$$
\alpha \leq I_{s}(x, \xi) \leq \beta
$$

where $\beta$ is a constant and $\alpha$ is a constant such that

$$
\alpha>\frac{-\eta}{1-\eta}
$$

Indeed, under conditions (41) and (42), (i) $A_{s}^{\eta}$ is continuous and coercive and therefore $\left(\mathrm{P}^{\eta, s}\right)$ is well posed, and (ii) the solution $u^{\eta, s}$ strongly converges with $s$ towards the solution $u^{\eta}$ of problem $\left(\mathrm{P}^{\eta}\right)$. This convergence result is proved in appendix $\mathrm{A}$.

Let us note that in problem $\left(\mathrm{P}^{\eta, s}\right)$, we use a prolongation $f^{\eta}$ of $f$ on $\Omega^{\square} \times \Xi$ and the sequence $\left\{u^{\eta, s}\right\}_{s \geq 1}$ depends on this prolongation. For a fixed $\eta$, the convergence of the sequence $u^{\eta}$ is guaranteed whatever the choice of prolongation. However, the convergence properties may be influenced by this choice. In appendix $\mathrm{A}$, it is given a sufficient condition on the prolongation $f^{\eta}$ in order to obtain convergence properties only dependent on the convergence of the sequence of approximate indicator functions $\left\{I_{s}\right\}_{s \geq 1}$.

\subsection{Separated representations of $I_{s}$}

We now address the problem of the construction of a separated representation (38) of $I_{s}$ verifying (41). We present two alternatives. The first one is a classical Karhunen-Loève expansion, for which restrictive assumptions on $I$ are required. The second one is an original definition of a constrained separated representation, which could be considered as a generalization of KarhunenLoève decomposition in order to impose uniform boundedness constraints.

\subsubsection{Karhunen-Loève expansion of the indicator function}

Separated representation $I_{s}$ can be defined as a classical Karhunen-Loève expansion of $I$, defined by

$$
\left\|I-I_{s}\right\|_{\Omega^{\square}}^{2}=\min _{\substack{g_{1}, \ldots, g_{s} \in L^{2}\left(\Omega^{\square}\right) \\ \chi_{1}, \ldots, \chi_{s} \in L_{P_{\xi}}^{2}(\Xi)}}\left\|I-\sum_{i=1}^{s} g_{i} \chi_{i}\right\|_{\Omega^{\square}}^{2}
$$

where $\|\cdot\|_{\Omega^{\square}}$ is the usual norm on $L_{\mu}^{2}\left(\Omega^{\square} \times \Xi\right)$, defined by

$$
\|I\|_{\Omega^{\square}}^{2}=\int_{\Omega^{\square} \times \Xi} I^{2} d \mu
$$


We denote $c_{I}: \overline{\Omega^{\square} \times \Omega^{\square}} \rightarrow \mathbb{R}$ the correlation kernel of $I$, defined by

$$
c_{I}\left(x, x^{\prime}\right)=\int_{\Xi} I(x, y) I\left(x^{\prime}, y\right) d P_{\xi}(y)=\mathbb{E}\left(I(x, \xi) I\left(x^{\prime}, \xi\right)\right)
$$

Functions $g_{i}$ are solutions of the eigenproblem

$$
\int_{\Omega^{\square}} c_{I}\left(x, x^{\prime}\right) g_{i}\left(x^{\prime}\right) d x^{\prime}=\gamma_{i} g_{i}(x)
$$

The eigenfunctions $\left\{g_{i}\right\}_{i \geq 1}$ form a basis of $L_{\mu}^{2}\left(\Omega^{\square} \times \Xi\right)$ and are orthogonal with respect to the natural inner product in $L_{\mu}^{2}$. If the $g_{i}$ are normalized, the associated functions $\chi_{i}$ are obtained by

$$
\chi_{i}(y)=\int_{\Omega^{\square}} I(x, y) g_{i}(x) d x
$$

The sequence $I_{s}$ converges towards $I$ in $L_{\mu}^{2}$ but this does not necessarily guarantee Property (41). However, under suitable restrictive assumptions on function $I$, discussed in appendix $\mathrm{B}$, we have a uniform convergence of $I_{s}$ towards I, i.e. ${ }^{13}$

$$
\lim _{s \longrightarrow \infty}\left\|I-I_{s}\right\|_{L_{P_{\xi}}^{\infty}\left(\Xi ; L^{\infty}\left(\Omega^{\square}\right)\right)}=0
$$

which means that for $\epsilon>0$, there exists $s^{\prime}(\epsilon)$ such that for $s \geq s^{\prime}(\epsilon)$, we have (almost everywhere and almost surely)

$$
\left|I-I_{s}\right| \leq \epsilon \quad \Rightarrow \quad-\epsilon \leq I_{s} \leq 1+\epsilon
$$

which implies the desired property (41) and (42) for $\epsilon<\frac{\eta}{1-\eta}$. Using a classical Karhunen-Loève expansion of $I$ is then theoretically possible, at the condition of choosing a rank sufficiently high in order to satisfy property (41). However, in practice, the $L^{\infty}$ convergence is very slow and a very high rank is required in order to reach a precision $\epsilon<\frac{\eta}{1-\eta}$ when using small values of $\eta$ (as illustrated below).

\subsubsection{Constrained separated representation of the indicator function}

We now introduce a new definition of separated representation (38) that tries to preserve the constraint (41) whatever the rank $s$. A detailed analysis of this new definition can be found in [24].

We propose to replace the definition (43) of the classical Karhunen-Loève expansion by the following definition. Starting from $I_{0}=0$, we define for

$\overline{{ }^{13}\|I\|_{L_{P_{\xi}}^{\infty}\left(\Xi ; L^{\infty}\left(\Omega^{\square}\right)\right)}}=P_{\xi}-\operatorname{ess}_{\sup _{\xi \in \Xi}}\|I(x, \cdot)\|_{L^{\infty}\left(\Omega^{\square}\right)}$, with $P_{\xi}-\operatorname{ess}_{\sup _{\xi \in \Xi}} f(\xi)=$
$\inf \left\{a \in \mathbb{R} ; P_{\xi}(\{y \in \Xi ; f(y)>a\})=0\right\}$ 
$s \geq 1$

$$
\left(g_{s}, \chi_{s}\right) \in \arg \min _{\substack{g \in L^{2}\left(\Omega^{\square}\right) \\ \chi \in L_{P_{\xi}}^{2}(\Xi)}} \frac{1}{2}\left\|I-I_{s-1}-g \chi\right\|_{\Omega^{\square}}^{2}+F\left(I_{s-1}+g \chi\right)
$$

where $F(I)$ is a suitable convex lower semi-continuous coercive functional on $V=L^{2}\left(\Omega^{\square}\right) \otimes L_{P_{\xi}}^{2}(\Xi)$ which takes high values for non admissible functions $I$, i.e. which do not verify (41).

Let us introduce the functional $J: V \rightarrow \mathbb{R} \cup\{+\infty\}$ defined by $J(z)=\frac{1}{2} \| I-$ $z \|_{\Omega^{\square}}^{2}+F(z)$. From properties of $F$ and of the norm $\|\cdot\|_{\Omega^{\square}}, J$ is a lower semicontinuous strictly convex and coercive functional on $V$. Let $\mathcal{R}_{1}=\{g \chi ; g \in$ $\left.L^{2}\left(\Omega^{\square}\right), \chi \in L_{P_{\xi}}^{2}(\Xi)\right\} \subset V$ be the set of rank-one separated functions in tensor product Hilbert space $V$. Equation (45) then defines $z_{s}=g_{s} \chi_{s} \in \mathcal{R}_{1}$ as follows

$$
J\left(I_{s-1}+z_{s}\right)=\min _{z \in \mathcal{R}_{1}} J\left(I_{s-1}+z\right)
$$

The existence of a minimizer $z_{s} \in \mathcal{R}_{1}$ classically follows from the properties of $J$ and from the fact that $\mathcal{R}_{1}$ is a weakly closed subset of $V$ (see [8]). In fact, the proposed definition of the decomposition $I_{s}$ is a particular case of the PGD for a class of nonlinear convex problems, whose mathematical analysis will be found in a forthcoming paper.

4.2.2.1 Choice of functional $F$ For the present context, we choose

$$
F(I)=\int_{\Omega^{\square} \times \Xi} f(I(x, y)) d \mu(x, y)
$$

where $f: \mathbb{R} \rightarrow \mathbb{R} \cup\{+\infty\}$ is a convex lower semi-continuous coercive function which is zero inside the admissible interval $[\alpha, \beta]$ and takes high values outside this interval. Ideally, we could choose

$$
f(x)= \begin{cases}0 & \text { if } x \in[\alpha, \beta] \\ +\infty & \text { if } x \notin[\alpha, \beta]\end{cases}
$$

but in practice, we select a two times differentiable regularization of this function:

$$
f(x)=\epsilon^{-1}\left(\max (x-\beta, 0)^{2}+\max (\alpha-x, 0)^{2}\right)
$$

4.2.2.2 Algorithm In order to compute a minimizer $z_{s}=g_{s} \chi_{s}$ of problem (46), we can use an alternated minimization procedure consisting in solving successively problems of type $\min _{g} J\left(I_{s-1}+g \chi\right)$ for a fixed $\chi$, and $\min _{\chi} J\left(I_{s-1}+\right.$ 
$g \chi)$ for a fixed $g$. Each sub-problem is a nonlinear optimization problem whose Euler-Lagrange equation writes

$$
\begin{array}{ll}
<J^{\prime}\left(I_{s-1}+g \chi\right), g^{*} \chi>=0 & \forall g^{*}, \\
<J^{\prime}\left(I_{s-1}+g \chi\right), g \chi^{*}>=0 & \forall \chi^{*}
\end{array}
$$

where

$$
\begin{gathered}
<J^{\prime}\left(I_{s-1}+g \chi\right), g^{*} \chi^{*}>=\left(g \chi+I_{s-1}-I, g^{*} \chi^{*}\right)+<F^{\prime}\left(I_{s-1}+g \chi\right), g^{*} \chi^{*}> \\
=\int_{\Omega^{\square} \times \Xi}\left(g \chi+I_{s-1}-I\right) g^{*} \chi^{*} d \mu+\int_{\Omega^{\square} \times \Xi} f^{\prime}\left(I_{s-1}+g \chi\right) g^{*} \chi^{*} d \mu
\end{gathered}
$$

The above Euler-Lagrange equations are nonlinear equations which can be solved with a Newton method.

4.2.2.3 Algebraic version In practice, the indicator function is approximated on a finite dimensional basis:

$$
I(x, y)=\sum_{i=1}^{n} \sum_{j=1}^{p} I_{i j} \varphi_{i}(x) \psi_{j}(y) .
$$

When selecting for $\varphi_{i}$ and $\psi_{j}$ interpolation bases, $I_{i j}$ corresponds to the value of the indicator function at a given point $x_{i}$ and for a given value $y_{j}$ of the parameter. We then transform the continuous point of view into a discrete point of view, consisting of a constrained singular value decomposition of matrix $\left(I_{i j}\right)$, defined by

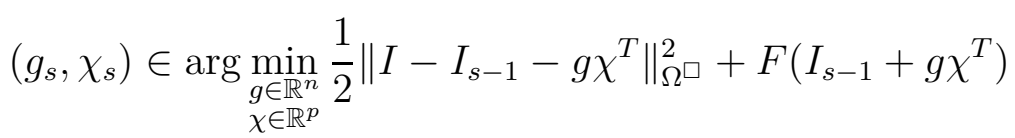

with $\|I\|_{\Omega^{\square}}^{2}=\sum_{i, j} I_{i j}^{2}$ and $F(I)=\sum_{i, j} f\left(I_{i j}\right)$. With this formulation, we try to constrain the values $\left(I_{s}\right)_{i j}$ to be inside the interval $[\alpha, \beta]$, which is equivalent to imposing the constraint (41) at the interpolation points.

4.2.2.4 An alternative definition Rather than the progressive definition (45), we could also adopt the following direct definition

$$
J\left(I_{s}\right)=\min _{\substack{g_{1}, \ldots, g_{s} \in L^{2}\left(\Omega^{\square}\right) \\ \chi_{1}, \ldots, \chi_{s} \in L_{P_{\xi}}^{2}(\Xi)}} J\left(\sum_{i=1}^{s} g_{i} \chi_{i}\right)
$$

which leads in practice to far better convergence properties of the sequence $I_{s}$. An alternated minimization procedure can also be used for the solution of this optimization problem. However, the construction of $I_{s}$ leads to higher computational costs than the progressive definition. 


\subsection{Illustration}

We consider here the same problem as in section 3.3 (with $\eta=0.01$ ). We choose a prolongation $f^{\eta}=1$ on the whole fictitious domain $\Omega^{\square}$. An approximation of the indicator function under the form (51) is introduced where we choose for $\left\{\varphi_{i}\right\}_{i=1}^{n}$ a linear finite element basis associated with the mesh of $\Omega^{\square}(n=1740)$ and for $\left\{\psi_{j}\right\}_{j=1}^{p}$ a piecewise linear interpolation basis associated with a uniform grid of $\Xi$ composed of 61 points $(p=61) . I_{i j}$ then corresponds to the value of the indicator function at a given point $x_{i}$ and for a given value $y_{j}$ of the parameter. In order to analyze the convergence of the separated representation of the indicator function, we introduce the following error indicator

$$
\varepsilon_{\Omega^{\square}}\left(I ; I^{*}\right)=\frac{\left\|I-I^{*}\right\|_{\Omega^{\square}}}{\left\|I^{*}\right\|_{\Omega^{\square}}}
$$

The solution is here searched in the approximation space introduced for the approximated indicator function, yielding $N=n=1740$ and $P=p=61$.

\subsubsection{Unconstrained separated representation and ill-posed problems}

We denote by $I_{s}$ the classical (unconstrained) separated representation of $I$. $u^{\eta}$ and $u^{\eta, s}$ then respectively denote the solutions of problem $\left(\mathrm{P}^{\eta}\right)$ with $I$ and of problem $\left(\mathrm{P}^{\eta, s}\right)$ with $I_{s}$. Figure 11(a) illustrates the convergence of the unconstrained decomposition $I_{s}$ with $s$ and Figure 11(b) shows the maximum and minimum values of $I_{s}$, along with the critical value $\frac{-\eta}{1-\eta}$. Note that in this finite dimensional case, the separated representation $I_{s}$ is equal to $I$ for $s \geq \min (n, p)=61$. We observe that the condition $I_{s}(x, \xi)>\frac{-\eta}{1-\eta}$ is not verified for any $s<61$. Therefore, the associated problem $\left(\mathrm{P}^{\eta, s}\right)$ is ill-posed for $s<61$. We observe the impact of this ill-posedness in Figure 12, which illustrates the erratic behavior of the sequence $u^{\eta, s} \in \mathcal{V}_{N}^{\square} \otimes \mathcal{S}_{P}$ (huge difference between consecutive solutions $u^{\eta, s}$ and $u^{\eta, s+1}$ associated with very close functions $I_{s}$ and $I_{s+1}$ ). Let us emphasize that in a continuous framework (in $\mathcal{V}^{\square} \otimes \mathcal{S}$ ), when replacing $I$ by $I_{s}$, the solution $u^{\eta, s}$ does not even exist. If we use the PGD method to compute the solution of problem $\left(\mathrm{P}^{\eta, s}\right)$ using $I_{s}$ with $s=20<61$ such that the problem is ill-posed, we observe a divergence of the PGD, as illustrated in Figure 13. 


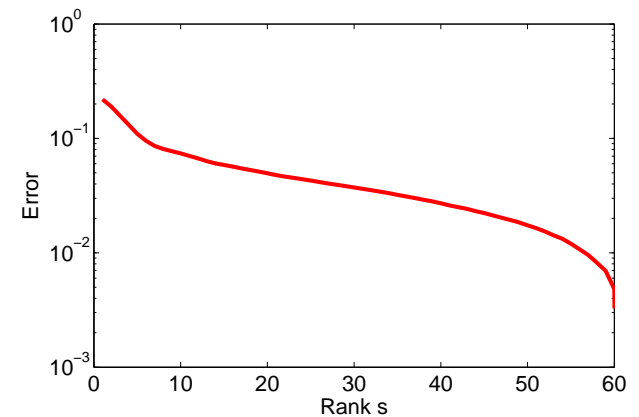

(a) $\varepsilon_{\Omega^{\square}}\left(I_{s} ; I\right)$

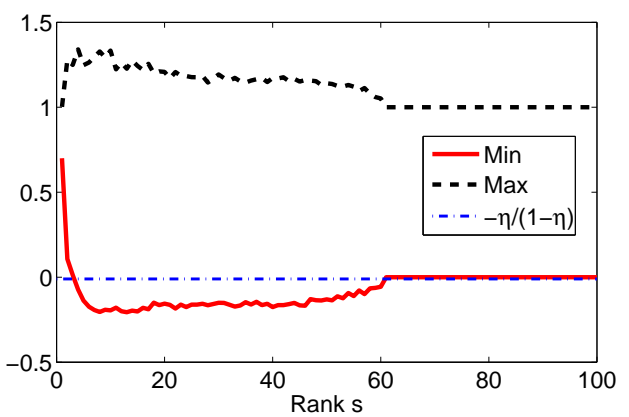

(b) $\min \left(I_{s}\right), \max \left(I_{s}\right)$

Figure 11. Unconstrained separation: evolution of the error indicator $\varepsilon_{\Omega^{\square}}\left(I_{s} ; I\right)$ with $s$ (a) and minimum and maximum values of $I_{s}(\mathrm{~b})$.

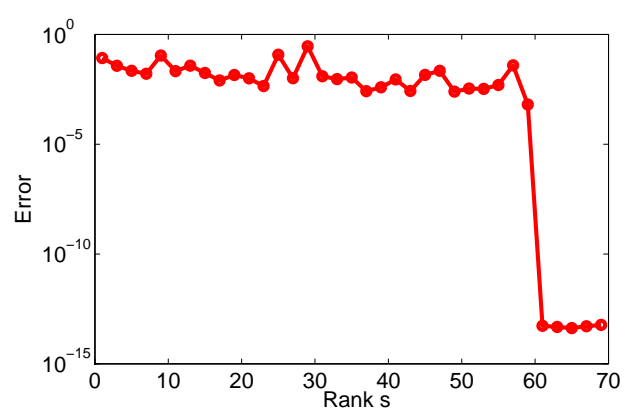

Figure 12. Unconstrained separation: error in solution $\varepsilon_{\Omega}\left(u^{\eta, s} ; u^{\eta}\right)$ with respect to the rank $s$ of $I_{s}$.

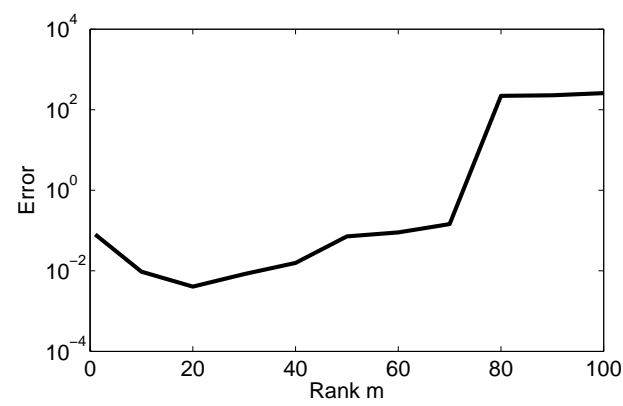

Figure 13. Unconstrained separation: evolution of the error $\varepsilon_{\Omega}\left(u_{m} ; u^{\eta}\right)$ with $m$ where $u_{m}$ is the rank- $m$ progressive PGD with $I$ replaced by $I_{s}$ with $s=20$. 


\subsubsection{Constrained separation}

We now consider the constrained separated representation $I_{s}$ of $I$ with a functional $F$ defined by (47) and (48), and depending on a parameter $\epsilon>0$. We fix the desired lower and upper bounds to $\alpha=0$ and $\beta=1$. Figure 14 shows the $L^{2}$ convergence of $I_{s}$ towards $I$, for different values of $\epsilon$. We note that the increase of $\epsilon$ results in a slower convergence of $I_{s}$. However, as illustrated in Figure 15, the constrained separated representation verifies the desired constraints more and more rapidly as $\epsilon$ is increased. When using $\epsilon=1000$ (resp. $\epsilon=200$ ), the condition $I_{s}(x, \xi)>\frac{-\eta}{1-\eta}$ is satisfied for $s>9$ (resp. $s>41$ ). A well-posed problem can then be obtained with a low rank approximation $I_{s}$ by choosing a sufficiently high parameter $\epsilon$.

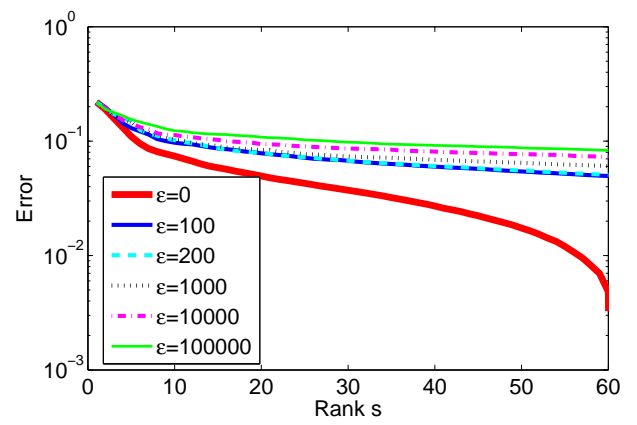

Figure 14. Constrained separation: evolution of the error indicator $\varepsilon_{\Omega^{\square}}\left(I_{s} ; I\right)$ with $s$ for different values of $\epsilon$.

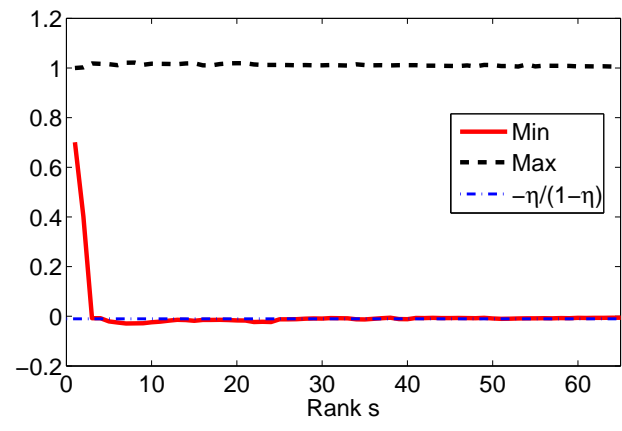

(a) $\epsilon=200$

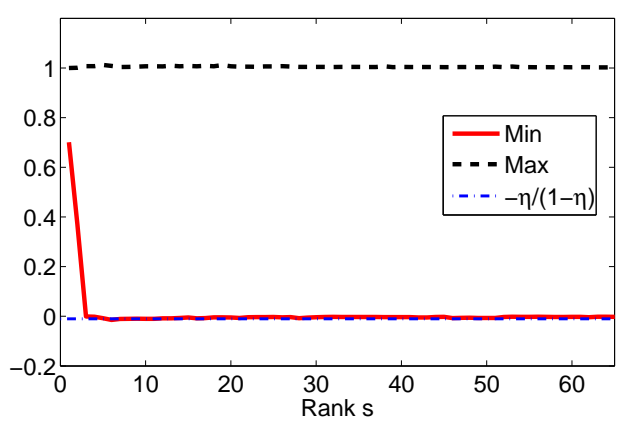

(b) $\epsilon=1000$

Figure 15. Constrained separation: minimum and maximum values of $I_{s}$ for $\epsilon=200$ (a) and $\epsilon=1000$ (b). 


\subsubsection{Regularization of the indicator function}

We now introduce a regularized version $I^{r}$ of the indicator function $I$, and we denote the associated solution of $\left(\mathrm{P}^{\eta}\right)$ by $u^{\eta, r}$. The aim of this regularization is to improve the convergence properties of separated representations, as discussed in appendix B. We define the regularization by $I^{r}=F^{r}(I)$, where $F^{r}$ is a truncated sine function defined by:

$$
F^{r}(x)=\left\{\begin{array}{rc}
\frac{1}{2}\left(1+\sin \left(\frac{\pi x}{2 l}\right)\right) & \text { if }-l \leq x \leq l \\
0 & \text { si } x<-l \\
1 & \text { si } x>l
\end{array}\right.
$$

Figure 16 shows the error in solution $\varepsilon_{\Omega}\left(u^{\eta, r} ; u^{\eta}\right)$ with respect to parameter $l$ of function $F^{r}$. We now select $l=0.1$, which corresponds to an error $\varepsilon_{\Omega}\left(u^{\eta, r} ; u^{\eta}\right) \leq 10^{-2}$. The rank-s separated representation of $I^{r}$ is denoted by $I_{s}^{r}$, and the corresponding solution is denoted by $u^{\eta, r, s}$ (solution of $\left(\mathrm{P}^{\eta, s}\right)$ with $I_{s}$ replaced by $I_{s}^{r}$ ). Figure 17 illustrates the significant improvement of the

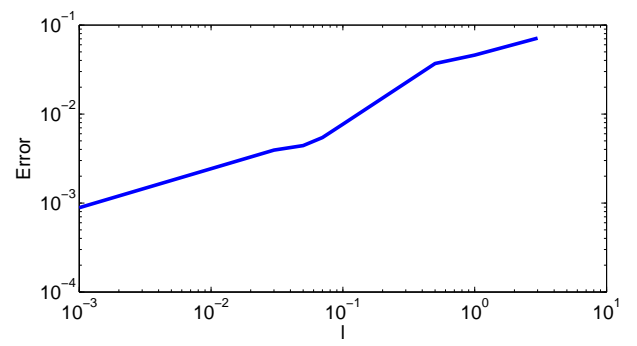

Figure 16. Regularized indicator function. Error $\varepsilon_{\Omega}\left(u^{\eta, r} ; u^{\eta}\right)$ with respect to $l$.

convergence with $s$ of the separated representations (unconstrained or constrained with $\epsilon=1000$ ) when introducing the regularization of the indicator function. Figure 18 shows the maximum and minimum values of the separated representations $I_{s}^{r}$. We observe that for the unconstrained decomposition, the condition $I_{s}^{r}(x, \xi)>\frac{-\eta}{1-\eta}$ is satisfied for $s>22$. Let us recall that without regularization, this condition was not satisfied for $s<61$. As regards the constrained decomposition, the condition is satisfied for a low rank $s=9$. From this study, we conclude that the use of the regularization combined with the constrained separated representation allows us to obtain (i) a good convergence of the separated representations $I_{s}^{r}$ and (ii) a well-posed problem with a very low rank decomposition.

Hereafter we use a constrained separation $I_{s}^{r}$ of the regularized indicator function $I^{r}$ using $\epsilon=1000$ and $l=0.1$. Figure 19 shows the convergence with $s$ of $u^{\eta, r, s}$ towards $u^{\eta, r}$. For $s=39$, we obtain an error $\varepsilon_{\Omega}\left(u^{\eta, r, s} ; u^{\eta, r}\right)$ lower than $10^{-2}$. 


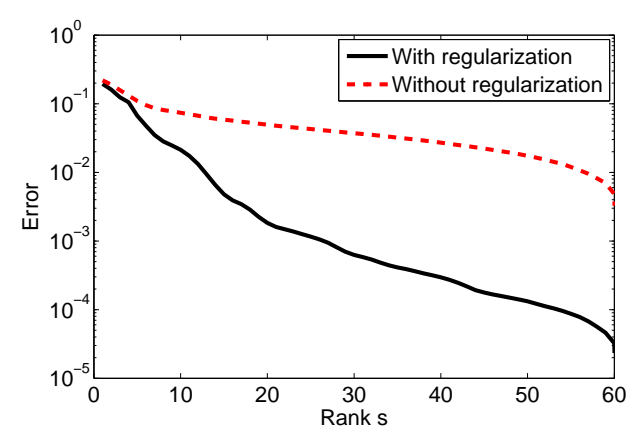

(a) Unconstrained

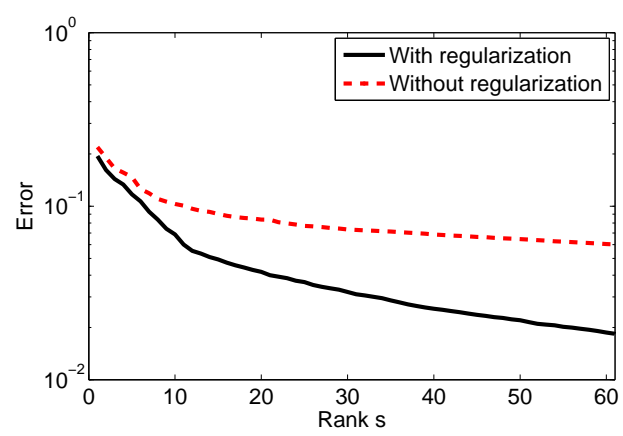

(b) Constrained

Figure 17. Influence of the regularization $(l=0.1)$ on the convergence of separated representations: error indicators $\varepsilon_{\Omega^{\square}}\left(I_{s} ; I\right)$ and $\varepsilon_{\Omega^{\square}}\left(I_{s}^{r} ; I^{r}\right)$ for unconstrained separations (a) and constrained separations with $\epsilon=1000$ (b).

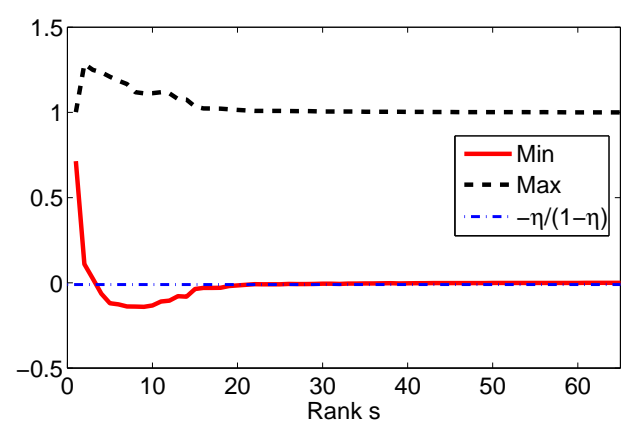

(a)

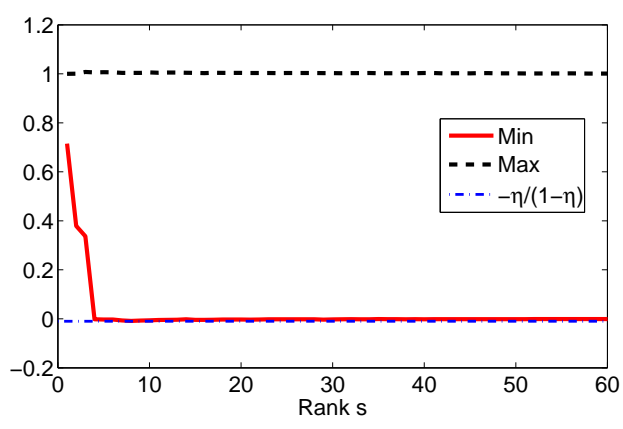

(b)

Figure 18. Minimum and maximum values of $I_{s}^{r}$ for unconstrained decomposition (a) and constrained decomposition with $\epsilon=1000$ (b).

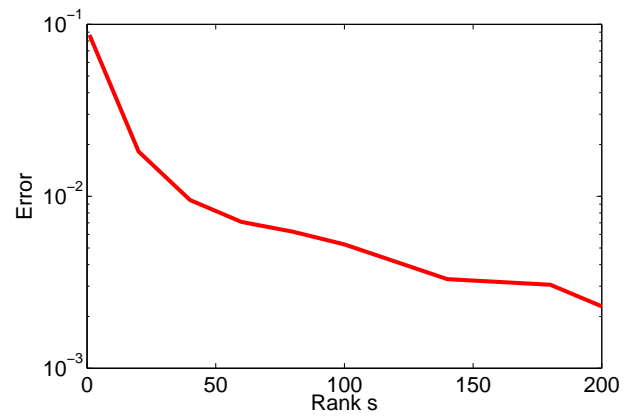

Figure 19. Error indicator $\varepsilon_{\Omega}\left(u^{\eta, r, s} ; u^{\eta, r}\right)$ with respect to $s$, where $u^{\eta, r, s}$ is the solution associated with a constrained separated representation $I_{s}^{r}$ (with $\epsilon=1000$ ) of the regularized function $I^{r}$ (with $l=0.1$ ). 


\subsubsection{Application of the PGD}

We now apply the progressive PGD algorithm to problem $\left(\mathrm{P}^{\eta, s}\right)$ with $I_{s}^{r}$, a separated representation of a regularized version $I^{r}$ of $I$. We use a regularization parameter $l=0.1$ corresponding to an error $\varepsilon_{\Omega}\left(u^{\eta, r} ; u^{\eta}\right) \leq 10^{-2}$ and we further perform a constrained separated representation with $\epsilon=1000$ and rank $s=39$, which corresponds to an error $\varepsilon_{\Omega}\left(u^{\eta, r, s} ; u^{\eta, r}\right) \leq 10^{-2}$. This representation ensures the well-posedness of the problem associated with $u^{\eta, r, s}$. Henceforth, $u_{m}$ denotes the progressive PGD approximation of $u^{\eta, r, s}$, associated with the approximate indicator function $I_{s}^{r}$. Figure 20 illustrates the convergence with $m$ of $u_{m}$ towards $u^{\eta, r, s}$. We note that $\varepsilon_{\Omega}\left(u_{m} ; u^{\eta, r, s}\right) \leq 10^{-2}$ is satisfied with a very low rank $m=8$.

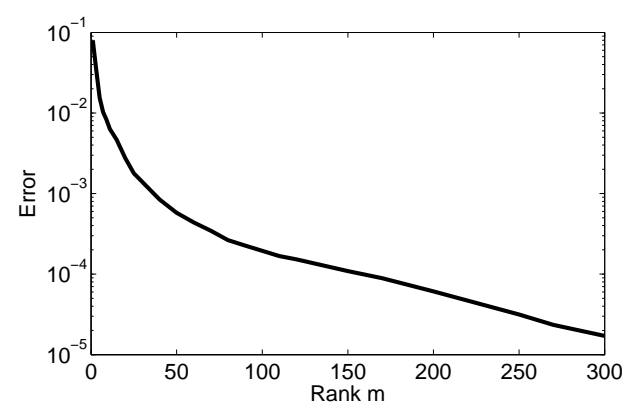

Figure 20. Convergence of the progressive PGD applied to the problem associated with the approximate indicator function $I_{s}^{r}$ (constrained separated representation of the regularized indicator function with $\epsilon=1000$ and $s=39$, and $l=0.1$ ): evolution of the error indicator $\varepsilon_{\Omega}\left(u_{m} ; u^{\eta, r, s}\right)$ with $m$. 


\section{Example : domain with two random sinusoïdal boundaries}

\subsection{Description of the problem}

The overall methodology is now conducted on the Poisson problem (2) with $f=1$ and a random domain $\Omega(\xi)$ delineated with two vertical lines and two random sinusoïdal curves (see Figure 21). The domain is characterized by

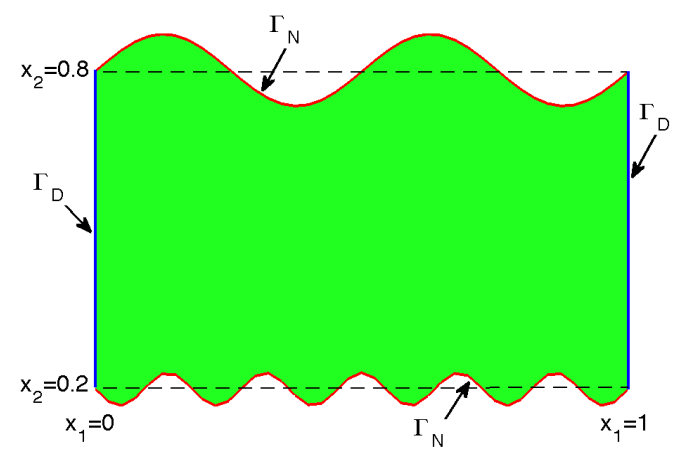

Figure 21. Domain with two random sinusoïdal boundaries: geometry and boundary conditions.

$$
\Omega(\xi)=\left\{x=\left(x_{1}, x_{2}\right) \in(0,1) \times \mathbb{R} ; f_{\text {inf }}\left(x_{1}, \xi_{2}\right)<x_{2}<f_{\text {sup }}\left(x_{1}, \xi_{1}\right)\right\}
$$

with

$$
\begin{aligned}
& f_{\text {sup }}\left(x_{1}, \xi_{1}\right)=0.8+0.2 \frac{\sin \left(12 \pi x_{1} \xi_{1}\right)}{6 \xi_{1}+1} \\
& f_{\text {inf }}\left(x_{1}, \xi_{2}\right)=0.2+0.2 \frac{\sin \left(12 \pi x_{1} \xi_{2}\right)}{6 \xi_{2}+1}
\end{aligned}
$$

and where $\xi_{1}$ and $\xi_{2}$ are two independent uniform random variables on $\Xi_{1}=$ $(0,1)$ and $\Xi_{2}=(0,1)$ respectively. The homogeneous Dirichlet boundary $\Gamma_{D}$ is composed of the vertical edges $x_{1}=0$ and $x_{1}=1$. The homogeneous Neumann boundary $\Gamma_{N}(\xi)$ is composed of the two sinusoidal curves $x_{2}=f_{\text {sup }}\left(x_{1}, \xi_{1}\right)$ and $x_{2}=f_{\text {inf }}\left(x_{1}, \xi_{1}\right)$.

We introduce a square fictitious domain $\Omega^{\square}=(0,1) \times(0,1)$. We choose for $\Gamma_{D}^{\square}$ the vertical edges $\{0\} \times(0,1)$ and $\{1\} \times(0,1)$, and for $\Gamma_{N}^{\square}$ the horizontal edges $(0,1) \times\{0\}$ and $(0,1) \times\{1\}$. The two sinusoidal curves are respectively characterized by the iso-zeros of two independent level-sets

$$
\phi_{1}\left(x, \xi_{1}\right)=x_{2}-f_{\text {sup }}\left(x_{1}, \xi_{1}\right), \quad \phi_{2}\left(x, \xi_{2}\right)=f_{\text {inf }}\left(x_{1}, \xi_{2}\right)-x_{2}
$$

and the domain $\Omega(\xi)$ is equivalently characterized by

$$
\Omega(\xi)=\left\{x \in \Omega^{\square} ; \phi(x, \xi)<0\right\}, \quad \phi(x, \xi)=\max \left\{\phi_{1}\left(x, \xi_{1}\right), \phi_{2}\left(x, \xi_{2}\right)\right\}
$$


We introduce a finite element approximation space $\mathcal{V}_{N}^{\square}$, with a regular mesh composed of 7200 triangular elements and $N=3721$ nodes, denoted $\left\{x_{i}\right\}_{i=1}^{N}$. The approximation space $\mathcal{S}_{P}$ is obtained by the tensorization of piecewise linear interpolation bases associated with uniform grids of $\Xi_{1}$ and $\Xi_{2}$, each grid containing 101 points. The resulting interpolation grid on $\Xi_{1} \times \Xi_{2}$ is composed of $P=101 \times 101=10201$ points, denoted $\left\{\xi^{k}\right\}_{k=1}^{P}$. We choose a parameter $\eta=0.01$ and a prolongation $f^{\eta}=1$ on $\Omega^{\square}$.

\subsection{Application of the PGD}

\subsubsection{Separated representation of the indicator function}

The indicator function of $\Omega(\xi)$ can be expressed as $I(x, \xi)=H(-\phi(x, \xi))$. If directly applied to this function, Karhunen-Loève decomposition (constrained or not) may present a very slow convergence. This is due to the statistical independence of the two sinusoidal boundaries. In order to decrease the dimensionality of the representation of $I$ (and therefore the rank of separated representations of bilinear and linear forms), we first split the domain $\Omega(\xi)$ into two subdomains $\Omega_{1}\left(\xi_{1}\right) \subset \Omega_{1}^{\square}=(0,1) \times(0.5,1)$ and $\Omega_{2}\left(\xi_{2}\right) \subset \Omega_{2}^{\square}=$ $(0,1) \times(0,0.5)$. In this way, we can introduce two independent indicator functions $I_{1}: \Omega_{1}^{\square} \times \Xi_{1} \rightarrow \mathbb{R}$ and $I_{2}: \Omega_{2}^{\square} \times \Xi_{2} \rightarrow \mathbb{R}$ associated with domains $\Omega_{1}\left(\xi_{1}\right)$ and $\Omega_{2}\left(\xi_{2}\right)$ respectively. Bilinear and linear forms are then obtained by splitting the integral on $\Omega^{\square}$ into integrals on $\Omega_{1}^{\square}$ and $\Omega_{2}^{\square}$. In order to improve convergence properties of their separated representations, the indicator functions are regularized as described in section 4.3.3. As regards the parameter $l$ of the regularization function $F^{r}$ introduced in (54), we propose to choose $l=\frac{L}{10}$, where $L$ is a characteristic length of the studied problem defined by

$$
L=\max _{i \in\{1 \ldots N\}}\left(\max _{j \in\{1 \ldots P\}} \phi\left(x_{i}, \xi^{j}\right)-\min _{j \in\{1 \ldots P\}} \phi\left(x_{i}, \xi^{j}\right)\right)
$$

where the $x_{i}$ are the nodes of the finite element mesh and where the $\xi^{j}$ are the nodes of the stochastic interpolation grid. For the problem concerned, $l$ is found 0.07 . It corresponds to an error $\varepsilon_{\Omega}\left(u^{\eta, r} ; u^{\eta}\right)=3.10^{-2}$. We then proceed to the computation of the two constrained rank-s separated representations $I_{1, s}^{r}$ and $I_{2, s}^{r}$ of the regularized functions $I_{1}^{r}$ and $I_{2}^{r}$ respectively, using $s=100$ and $\epsilon=1000$ for each of the two functions. With an abuse of notation, we denote by $I_{s}^{r}$ the resulting approximation of the indicator function $I$, and by $u^{\eta, r, s}$ the Galerkin approximation in $\mathcal{V}_{N}^{\square} \otimes \mathcal{S}_{P}$ of the problem $\left(\mathrm{P}^{\eta, s}\right)$, with $I_{s}$ replaced by $I_{s}^{r}$. 


\subsubsection{Construction of the PGD}

We can now construct the progressive PGD sequence $u_{m}$, which theoretically converges towards $u^{\eta, r, s} \in \mathcal{V}_{N}^{\square} \otimes \mathcal{S}_{P}$, which is considered as the reference solution ${ }^{14}$. Figure 22 illustrates the convergence of $u_{m}$ towards $u^{\eta, r, s}$. We note that an error $\varepsilon_{\Omega}\left(u_{m} ; \tilde{u}^{\eta, r, s}\right) \leq 3.10^{-2}$ is reached for a low rank $m=12$. Figures

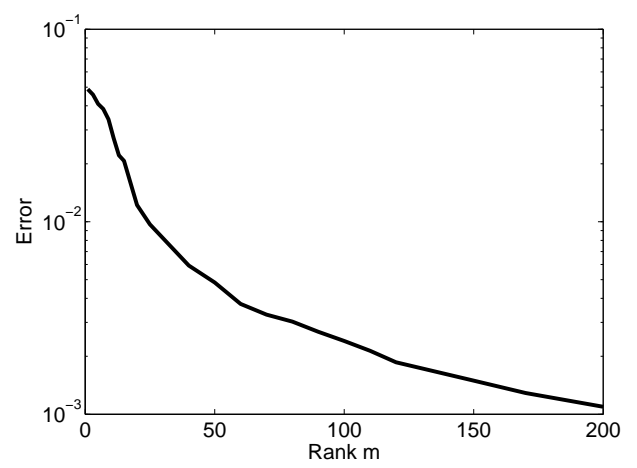

Figure 22. Convergence of PGD for the problem with approximate indicator function $I_{1, s}^{r}$ and $I_{2, s}^{r}$ (constrained separated representation with $\epsilon=1000$ and $s=100$ ). Evolution of the error indicator $\varepsilon_{\Omega}\left(u_{m} ; u^{\eta, r, s}\right)$ with $m$.

23 and 24 respectively show the first deterministic vectors $w_{i}$ and stochastic parametric functions $\lambda_{i}(\xi)$ of the PGD. They illustrate the progressive construction of the PGD from a global representation of the solution towards a refined representation of it.

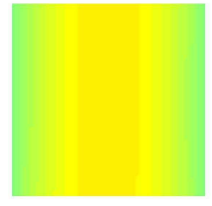

(a) $w_{1}$

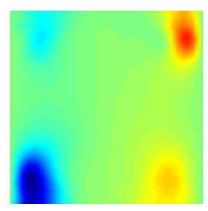

(e) $w_{5}$

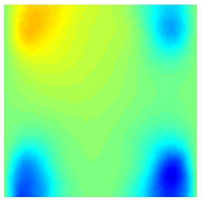

(b) $w_{2}$

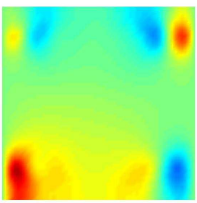

(f) $w_{6}$

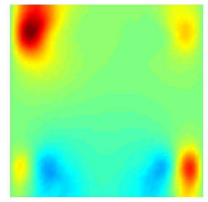

(c) $w_{3}$

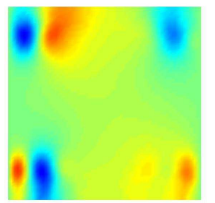

(g) $w_{7}$

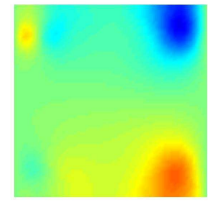

(d) $w_{4}$

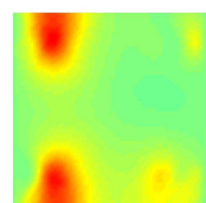

(h) $w_{8}$

Figure 23. First deterministic vectors $w_{i}$ of the PGD.

Remark 3 We notice a very high irregularity of parametric functions $\lambda_{i}(\xi)$, and therefore of $u_{m}(x, \xi)$ with respect to $\xi$. This is due to the particular stochastic parametrization of the sinusoidal curves. The capture of this irregularity

\footnotetext{
${ }^{14}$ In practice, we use as a reference solution a very good approximation of $u^{\eta, r, s}$, obtained with a high rank PGD approximation.
} 


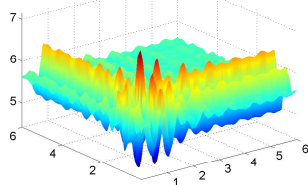

(a) $\lambda_{1}$

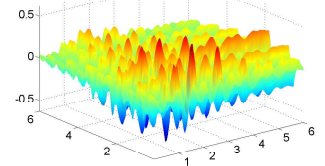

(b) $\lambda_{2}$

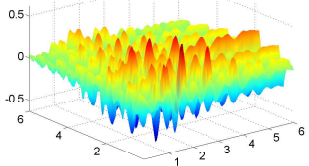

(c) $\lambda_{3}$

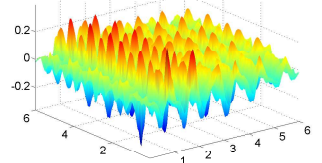

(d) $\lambda_{4}$
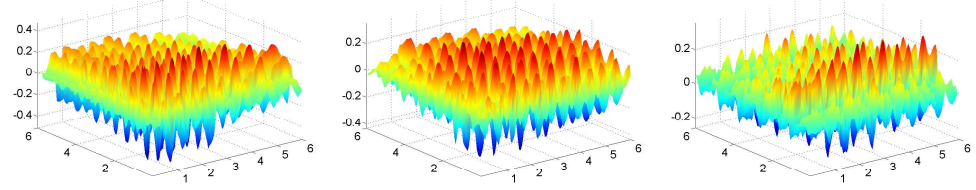

(e) $\lambda_{5}$

(f) $\lambda_{6}$

(g) $\lambda_{7}$

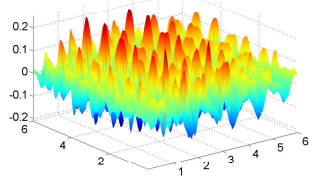

(h) $\lambda_{8}$

Figure 24. First stochastic parametric functions $\lambda_{i}\left(\xi_{1}, \xi_{2}\right)$ of the PGD.

in the solution requires the use of very high dimensional approximation spaces $\mathcal{S}_{P}$, which is made affordable by the use of the present methodology.

Figure 25 shows the reference solution $u^{\eta, r, s}$ and PGD approximations $u_{m}$ for different ranks $m$ and for a particular realization of $\xi$. Finally, Figure

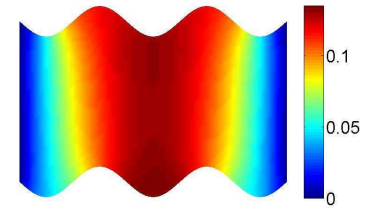

(a) $u^{\eta, r, s}$

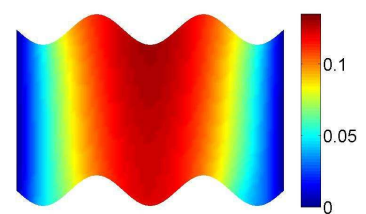

(d) $u_{5}$

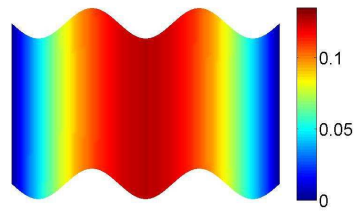

(b) $u_{1}$

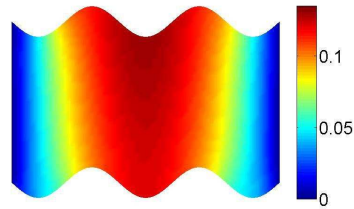

(e) $u_{9}$

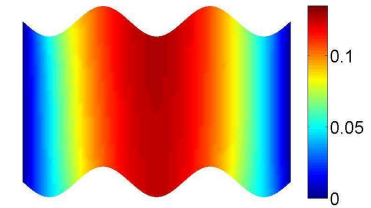

(c) $u_{3}$

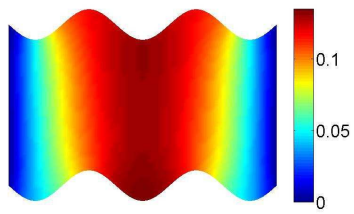

(f) $u_{60}$

Figure 25. Comparison between $u^{\eta, r, s}(\cdot, \xi)$ and $u_{m}(\cdot, \xi)$ for different $m$, and for $\xi=\left(\frac{5}{12}, \frac{5}{12}\right)$.

26 shows the reference solution $u^{\eta, r, s}$ and the PGD approximation $u_{60}$ for several realizations of $\xi$. We can draw the same conclusions as for the previous example. Indeed, the PGD gives an accurate description of the solution with a low rank separated representation. 


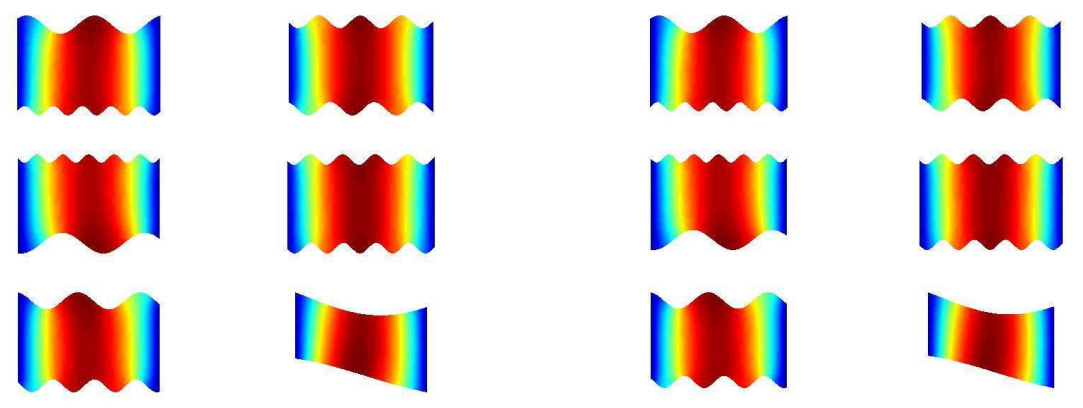

(a)

(b)

Figure 26. Comparison between $u^{\eta, r, s}(\cdot, \xi)$ (a) and $u_{60}(\cdot, \xi)$ (b) for several realizations of $\xi$. 


\section{Conclusion}

A numerical methodology has been proposed for the solution of partial differential equations defined on uncertain parameterized domains. This methodology first relies on a fictitious domain approach, yielding a formulation of the problem in a tensor product space. This tensor product structure is then exploited by applying the Proper Generalized Decomposition method, allowing the a priori construction of a tensor product approximation of the solution. This PGD method can be seen as a model reduction technique which automatically constructs reduced bases of space functions and parametric functions for an optimal separated representation of the solution. In order to make efficient the PGD algorithms, specific techniques are introduced for obtaining low rank separated representations of the indicator function of the domain, thus yielding low rank representations of variational forms involved in the weak formulation of the problem. In particular, smoothing of indicator functions is introduced in order to accelerate the convergence of their separated representations. Moreover, a new constrained tensor product approximation is used in order to preserve some positivity constraints that ensure a well-posedness of problems associated with approximate indicator functions. All these ingredients have been put together in a complete methodology, the efficiency and accuracy of which have been illustrated on examples. Future works will be devoted to the extension of this methodology to high dimensional parametric problems (by exploiting multidimensional separated representation methods), with specific applications to shape optimization and shape identification, where the shape is controlled by multiple parameters.

\section{A Convergence of the fictitious domain solutions associated with approximate indicator functions}

In this section, we give two convergence results.

(i) The convergence with $\eta$ of the solution $u^{\eta}$ of problem $\left(\mathrm{P}^{\eta}\right)$ towards the solution $u$ of the initial problem $(\mathrm{P})$

(ii) The convergence of the sequence of solutions $u^{\eta, s}$ of problems $\left(\mathrm{P}^{\eta, s}\right)$, associated with rank-s separated representations $I_{s}$ of the indicator function $I$, towards the solution $u^{\eta}$ of problem $\left(\mathrm{P}^{\eta}\right)$

The first result is a variant of the proof given in [11] in the deterministic context, with an improved estimation of the rate of convergence. The second convergence result is proved under a strong assumption on the sequence $I_{s}$, which is discussed in section B. 


\section{A.1 Convergence of solutions of regularized problems}

We first introduce the following decomposition of the bilinear form $A^{\eta}$ :

$$
A^{\eta}(u, v)=A(u, v)+\eta C(u, v)=(1-\eta) A(u, v)+\eta B(u, v)
$$

with

$$
\begin{aligned}
& C(u, v)=\int_{\Xi} \int_{\Omega^{\square} \backslash \Omega} \nabla u \cdot \nabla v d \mu, \\
& B(u, v)=\int_{\Xi} \int_{\Omega^{\square}} \nabla u \cdot \nabla v d \mu
\end{aligned}
$$

We recall that the norm $\|\cdot\|_{\mathcal{W}}$ in $\mathcal{W}^{\square}=\mathcal{V}^{\square} \otimes \mathcal{S}$ is defined by

$$
\|v\|_{\mathcal{W}^{\square}}^{2}=\int_{\Xi} \int_{\Omega^{\square}}|\nabla v|^{2} d \mu=B(v, v)
$$

We introduce the following space

$$
\mathcal{X}^{\square}=L_{P_{\xi}}^{2}\left(\Xi ; H_{0}^{1}\left(\Omega^{\square} \backslash \Omega\right)\right) \subset \mathcal{W}^{\square}
$$

and we define the following subspace of $\mathcal{W}^{\square}$ :

$$
\mathcal{W}_{0}^{\square}=\left\{v \in \mathcal{W}^{\square} ; C(v, w)=0 \forall w \in \mathcal{X}^{\square}\right\}
$$

$\mathcal{W}_{0}^{\square}$ corresponds to functions in $\mathcal{W}^{\square}$ which are harmonic in the non-physical domain $\Omega^{\square} \backslash \Omega$. There exists a unique function $\hat{u}$ such that

$$
\hat{u} \in \mathcal{W}_{0}^{\square} \quad \text { and } \quad \hat{u}(\cdot, y)=u(\cdot, y) \quad \text { on } \quad \Omega(y)
$$

$\hat{u}$ is the unique harmonic prolongation of the solution $u$ of $(\mathrm{P})$.

Theorem 2 When $\eta \rightarrow 0$, the solution $u^{\eta}$ of problem $\left(\mathrm{P}^{\eta}\right)$ converges towards $\hat{u}$ in the following sense:

$$
\lim _{\eta \rightarrow 0}\left\|\hat{u}-u^{\eta}\right\|_{\mathcal{W} \square}=0,
$$

and converges to the solution $u$ of problem $(\mathrm{P})$ in the following sense

$$
\limsup _{\eta \rightarrow 0} \eta^{-1}\left\|u-u^{\eta}\right\|_{\mathcal{W}}<\infty
$$

or again

$$
\lim _{\eta \rightarrow 0} \eta^{-\delta}\left\|u-u^{\eta}\right\|_{\mathcal{W}}=0 \quad \forall \delta<1
$$

which indicates that we have a linear convergence with $\eta$ when we only consider the physical part of the solution (restriction to the physical domain $\Omega$ ). 
Proof. We have

$$
\begin{aligned}
A^{\eta}\left(\hat{u}-u^{\eta}, \hat{u}-u^{\eta}\right) & =A^{\eta}\left(\hat{u}, \hat{u}-u^{\eta}\right)-A^{\eta}\left(u^{\eta}, \hat{u}-u^{\eta}\right) \\
& =L\left(\hat{u}-u^{\eta}\right)+\eta C\left(\hat{u}, \hat{u}-u^{\eta}\right)-L\left(\hat{u}-u^{\eta}\right) \\
& =\eta C\left(\hat{u}, \hat{u}-u^{\eta}\right)
\end{aligned}
$$

Using

$$
A^{\eta}(v, v)=(1-\eta) A(v, v)+\eta B(v, v)=(1-\eta)\|v\|_{\mathcal{W}}^{2}+\eta\|v\|_{\mathcal{W} \square}^{2}
$$

we first obtain

$$
(1-\eta)\left\|\hat{u}-u^{\eta}\right\|_{\mathcal{W}}^{2}+\eta\left\|\hat{u}-u^{\eta}\right\|_{\mathcal{W} \square}^{2}=\eta C\left(\hat{u}, \hat{u}-u^{\eta}\right)
$$

and also

$$
\begin{aligned}
(1-\eta)\left\|\hat{u}-u^{\eta}\right\|_{\mathcal{W}}^{2}+\eta\left\|\hat{u}-u^{\eta}\right\|_{\mathcal{W} \square}^{2} & \leq \eta C(\hat{u}, \hat{u})^{1 / 2} C\left(\hat{u}-u^{\eta}, \hat{u}-u^{\eta}\right)^{1 / 2} \\
& \leq \eta B(\hat{u}, \hat{u})^{1 / 2} B\left(\hat{u}-u^{\eta}, \hat{u}-u^{\eta}\right)^{1 / 2} \\
& \leq \eta\|\hat{u}\|_{\mathcal{W} \square}\left\|\hat{u}-u^{\eta}\right\|_{\mathcal{W} \square}
\end{aligned}
$$

We first deduce that

$$
\left\|\hat{u}-u^{\eta}\right\|_{\mathcal{W} \square} \leq\|\hat{u}\|_{\mathcal{W} \square}
$$

which proves that the sequence $u^{\eta}$ is bounded. Therefore, we can extract a subsequence, still denoted $u^{\eta}$ that converges weakly to an element $u^{*} \in \mathcal{W}^{\square}$. $u^{\eta}$ verifies

$$
A\left(u^{\eta}, v\right)+\eta C\left(u^{\eta}, v\right)=L(v) \quad \forall v \in \mathcal{W}^{\square}
$$

Thus, taking the limit with $\eta \rightarrow 0$, we obtain

$$
A\left(u^{*}, v\right)=L(v) \quad \forall v \in \mathcal{W}^{\square}
$$

which proves that the restriction of $u^{*}$ to the domain $\Omega$ coincides with the solution $u$. Moreover, for all $\eta$, we have

$$
C\left(u^{\eta}, v\right)=0 \quad \forall v \in \mathcal{X}^{\square}
$$

and taking the limit with $\eta$, we obtain that

$$
C\left(u^{*}, v\right)=0 \quad \forall v \in \mathcal{X}^{\square}
$$

which gives $u^{*} \in \mathcal{W}_{0}^{\square}$. Since the unique prolongation of $u$ in $\mathcal{W}_{0}^{\square}$ is $\hat{u}$, we have $u^{*}=\hat{u}$. By uniqueness of the limit, we therefore obtain that the whole sequence $\left\{u^{\eta}\right\}_{\eta}$ weakly converges to $\hat{u}$. From (A.3), we have

$$
\frac{1-\eta}{\eta}\left\|u-u^{\eta}\right\|_{\mathcal{W}}^{2}+\left\|\hat{u}-u^{\eta}\right\|_{\mathcal{W} \square}^{2}=C\left(\hat{u}, \hat{u}-u^{\eta}\right) \stackrel{\eta \rightarrow 0}{\longrightarrow} 0
$$


and we obtain that

$$
\lim _{\eta \rightarrow 0}\left\|\hat{u}-u^{\eta}\right\|_{\mathcal{W} \square}=0
$$

and also that

$$
\lim _{\eta \rightarrow 0} \eta^{-1 / 2}\left\|u-u^{\eta}\right\|_{\mathcal{W}}=0
$$

In order to refine the convergence in the $\mathcal{W}$-norm, we note that $\hat{u}$ and $u^{\eta}$ belong to $\mathcal{W}_{0}^{\square}$, and that the norms $\|\cdot\|_{\mathcal{W}}$ and $\|\cdot\|_{\mathcal{W} \square}$ are equivalent on $\mathcal{W}_{0}^{\square}$. Therefore, there exists a constant $\gamma>0$ such that

$$
\left\|\hat{u}-u^{\eta}\right\|_{\mathcal{W} \square} \leq \gamma\left\|\hat{u}-u^{\eta}\right\|_{\mathcal{W}}
$$

Using this inequality and (A.4), we obtain

$\left\|\hat{u}-u^{\eta}\right\|_{\mathcal{W}}^{2} \leq\left\|\hat{u}-u^{\eta}\right\|_{\mathcal{W}}^{2}+\eta\left\|\hat{u}-u^{\eta}\right\|_{\mathcal{W} \square}^{2}-\eta\left\|\hat{u}-u^{\eta}\right\|_{\mathcal{W}}^{2} \leq \eta \gamma\|\hat{u}\|_{\mathcal{W} \square}\left\|\hat{u}-u^{\eta}\right\|_{\mathcal{W}}$

and therefore

$$
\frac{1}{\eta}\left\|\hat{u}-u^{\eta}\right\|_{\mathcal{W}} \leq \gamma\|\hat{u}\|_{\mathcal{W} \square}<\infty
$$

which yields (A.2).

Remark 4 Under sufficient regularity assumptions on the boundary on the domain $\Omega$, we have that $u^{\eta}$ verifies the following transmission condition on the internal boundary $\Gamma_{N}$ :

$$
\eta \frac{\partial u_{+}^{\eta}}{\partial n}-\frac{\partial u_{-}^{\eta}}{\partial n}=0
$$

where $n$ is the unit normal to $\Gamma_{N}$ and $u_{+}^{\eta}$ (resp. $u_{-}^{\eta}$ ) is the restriction of $u^{\eta}$ to $\Omega^{\square} \backslash \Omega$ (resp. $\Omega$ ). For a sufficiently small $\eta$, we therefore obtain an approximate verification of the homogeneous Neumann boundary condition $\frac{\partial u_{-}^{\eta}}{\partial n} \approx 0$.

\section{A.2 Convergence of solutions with approximate indicator functions}

We suppose that the sequence $\left\{I_{s}\right\}_{s>1}$ is such that for $s \geq s^{\prime}$, condition (41) is verified. The existence of such an $s^{\prime}$ is a consequence of the following stronger assumption on the sequence $I_{s}$, which is discussed in section B.

Assumption 3 We suppose that the sequence $\left\{I_{s}\right\}_{s \geq 1}$ uniformly converges towards I:

$$
\lim _{s \rightarrow \infty}\left\|I-I_{s}\right\|_{L_{P_{\xi}}^{\infty}\left(\Xi ; L^{\infty}\left(\Omega^{\square}\right)\right)}=0
$$


We denote by $\delta_{s}=\left\|I-I_{s}\right\|_{L_{P_{\xi}}^{\infty}\left(\Xi ; L^{\infty}\left(\Omega^{\square}\right)\right)}$. In the following, for any $\eta$, we only consider the sequence $\left\{I_{s}\right\}_{s \geq s^{\prime}(\eta)}$ such that

$$
\frac{1-\eta}{\eta} \delta_{s} \leq \gamma<1
$$

with some constant $\gamma$. Note that condition (A.6) implies that $I_{s} \geq-\delta_{s} \geq$ $-\gamma \frac{\eta}{1-\eta}>-\frac{\eta}{1-\eta}$, which is the ellipticity condition (42). For a domain $\omega$, eventually random, we denote by $\|\cdot\|_{\omega}$ the norm in $L_{P_{\xi}}^{2}(\Xi ; \omega)$, defined by

$$
\|v\|_{\omega}^{2}=\int_{\Xi} \int_{\omega} v^{2} d \mu
$$

Theorem 4 Under Assumption 3, we have the strong convergence of the sequence $\left\{u^{\eta, s}\right\}_{s \in \mathbb{N}}$, with $u^{\eta, s}$ the solution of $\left(\mathrm{P}^{\eta, s}\right)$, towards the solution $u^{\eta}$ of $\left(\mathrm{P}^{\eta}\right)$ :

$$
\lim _{s \rightarrow \infty}\left\|u^{\eta}-u^{\eta, s}\right\|_{A^{\eta}}=0
$$

More precisely,

$$
\left\|u^{\eta}-u^{\eta, s}\right\|_{A^{\eta}} \leq G(s, \eta) D(\eta)
$$

where $G(s, \eta) \rightarrow 0$ as $s \rightarrow \infty$ and $G(s, \eta)$ uniformly bounded with $\eta$, and where $D(\eta)$ is a constant which depends on the prolongation $f^{\eta}$ of $f$ :

$$
D(\eta)=\left(\|f\|_{\Omega}^{2}+\left\|f^{\eta} / \sqrt{\eta}\right\|_{\Omega^{\square} \backslash \Omega}^{2}\right)^{1 / 2}(1+\eta)^{1 / 2}
$$

Proof. The bilinear form $A^{\eta}$, defined in (15), is continuous symmetric and coercive. It defines a norm in $\mathcal{W}^{\square}$, defined by $\|v\|_{A^{\eta}}^{2}=A^{\eta}(v, v)$, and such that $\eta\|\cdot\|_{\mathcal{W} \square}^{2} \leq\|\cdot\|_{A^{\eta}}^{2} \leq\|\cdot\|_{\mathcal{W}^{\square}}^{2}$. We have

$$
\begin{aligned}
\left\|u^{\eta}-u^{\eta, s}\right\|_{A^{\eta}}^{2}= & A^{\eta}\left(u^{\eta}-u^{\eta, s}, u^{\eta}-u^{\eta, s}\right) \\
= & A^{\eta}\left(u^{\eta}, u^{\eta}-u^{\eta, s}\right)-A^{\eta}\left(u^{\eta, s}, u^{\eta}-u^{\eta, s}\right) \\
= & L\left(u^{\eta}-u^{\eta, s}\right)-L_{s}^{\eta}\left(u^{\eta}-u^{\eta, s}\right) \\
& +A_{s}^{\eta}\left(u^{\eta, s}, u^{\eta}-u^{\eta, s}\right)-A^{\eta}\left(u^{\eta, s}, u^{\eta}-u^{\eta, s}\right) \\
= & \left(L-L_{s}^{\eta}\right)\left(u^{\eta}-u^{\eta, s}\right)+\left(A_{s}^{\eta}-A^{\eta}\right)\left(u^{\eta, s}, u^{\eta}-u^{\eta, s}\right)
\end{aligned}
$$

Noting that

$$
\|\nabla u\|_{\Omega^{\square}}^{2} \leq \frac{1}{\eta}\|u\|_{A^{\eta}}^{2}
$$

and introducing $\delta_{s}=\left\|I-I_{s}\right\|_{L_{P_{\xi}}^{\infty}\left(\Xi ; L^{\infty}\left(\Omega^{\square}\right)\right)}$, we obtain

$$
\begin{aligned}
\left(A_{s}^{\eta}-A^{\eta}\right)\left(u^{\eta, s}, u^{\eta}-u^{\eta, s}\right) & \leq(1-\eta) \delta_{s}\left\|\nabla u^{\eta, s}\right\|_{\Omega^{\square}}\left\|\nabla\left(u^{\eta}-u^{\eta, s}\right)\right\|_{\Omega^{\square}} \\
& \leq \delta_{s} \frac{1-\eta}{\eta}\left\|u^{\eta, s}\right\|_{A^{\eta}}\left\|u^{\eta}-u^{\eta, s}\right\|_{A^{\eta}}
\end{aligned}
$$


Let $\|\cdot\|_{A_{s}^{\eta}}$ be the norm on $\mathcal{W}^{\square}$ induced by $A_{s}^{\eta}$, defined by $\|v\|_{A_{s}^{\eta}}^{2}=A_{s}^{\eta}(v, v)$. We also have

$$
\begin{aligned}
\|u\|_{A_{s}^{\eta}}^{2} & =\|u\|_{A^{\eta}}^{2}+(1-\eta) \int_{\Omega^{\square}}\left(I_{s}-I\right) \nabla u^{2} \\
& \geq\|u\|_{A^{\eta}}^{2}-(1-\eta) \delta_{s}\|\nabla u\|_{\Omega^{\square}}^{2} \\
& \geq\|u\|_{A^{\eta}}^{2}\left(1-\frac{1-\eta}{\eta} \delta_{s}\right)
\end{aligned}
$$

where $\frac{1-\eta}{\eta} \delta_{s} \leq \gamma<1$ with assumption (A.6). We then have

$$
\begin{aligned}
& \left(1-\frac{1-\eta}{\eta} \delta_{s}\right)\left\|u^{\eta, s}\right\|_{A^{\eta}}^{2} \leq\left\|u^{\eta, s}\right\|_{A_{s}^{\eta}}^{2}=L_{s}^{\eta}\left(u^{\eta, s}\right) \\
& \leq\left(1+\delta_{s}\right)\left(\|f\|_{\Omega}\left\|u^{\eta, s}\right\|_{\Omega}+\left\|f^{\eta}\right\|_{\Omega^{\square} \backslash \Omega}\left\|u^{\eta, s}\right\|_{\Omega^{\square} \backslash \Omega}\right)
\end{aligned}
$$

We denote by $C_{\omega}$ the constant defined by

$$
C_{\omega}=\sup _{v} \frac{\|v\|_{\omega}}{\|\nabla v\|_{\omega}}
$$

and by $C=\max \left\{C_{\Omega}, C_{\Omega^{\square}}\right\}$ (constants $C_{\Omega}$ and $C_{\Omega^{\square}}$ are finite since $\partial \Omega$ and $\partial \Omega^{\square}$ possess a Dirichlet part). Then,

$$
\begin{aligned}
& \left(1-\frac{1-\eta}{\eta} \delta_{s}\right)\left\|u^{\eta, s}\right\|_{A^{\eta}}^{2} \\
& \leq\left(1+\delta_{s}\right) C\left(\|f\|_{\Omega}\left\|\nabla u^{\eta, s}\right\|_{\Omega}+\eta\left\|f^{\eta} / \eta\right\|_{\Omega^{\square} \backslash \Omega}\left\|\nabla u^{\eta, s}\right\|_{\Omega^{\square}}\right) \\
& \leq\left(1+\delta_{s}\right) C \sqrt{2}\left(\|f\|_{\Omega}^{2}+\eta\left\|f^{\eta} / \eta\right\|_{\Omega^{\square} \backslash \Omega}^{2}\right)^{1 / 2}\left(\left\|\nabla u^{\eta, s}\right\|_{\Omega}^{2}+\eta\left\|\nabla u^{\eta, s}\right\|_{\Omega^{\square}}^{2}\right)^{1 / 2} \\
& \leq\left(1+\delta_{s}\right) C \sqrt{2}\left(\|f\|_{\Omega}^{2}+\eta\left\|f^{\eta} / \eta\right\|_{\Omega^{\square} \backslash \Omega}^{2}\right)^{1 / 2}(1+\eta)^{1 / 2}\left\|u^{\eta, s}\right\|_{A^{\eta}}
\end{aligned}
$$

It yields

$$
\left\|u^{\eta, s}\right\|_{A^{\eta}} \leq \sqrt{2} \frac{1+\delta_{s}}{1-\frac{1-\eta}{\eta} \delta_{s}} C D(\eta)
$$

with $D(\eta)=(1+\eta)^{1 / 2}\left(\|f\|_{\Omega}^{2}+\left\|f^{\eta} / \sqrt{\eta}\right\|_{\Omega^{\square} \backslash \Omega}^{2}\right)^{1 / 2}$. In the same way, we obtain

$$
\begin{aligned}
\left(L-L_{s}^{\eta}\right)\left(u^{\eta}-u^{\eta, s}\right) & \leq \delta_{s}\left(\|f\|_{\Omega}\left\|u^{\eta}-u^{\eta, s}\right\|_{\Omega}+\left\|f^{\eta}\right\|_{\Omega^{\square} \backslash \Omega}\left\|u^{\eta}-u^{\eta, s}\right\|_{\Omega^{\square} \backslash \Omega}\right) \\
& \leq \delta_{s} C D(\eta) \sqrt{2}\left\|u^{\eta}-u^{\eta, s}\right\|_{A^{\eta}}
\end{aligned}
$$

Finally, using (A.8), (A.9), (A.10) and (A.11), we obtain

$$
\left\|u^{\eta}-u^{\eta, s}\right\|_{A^{\eta}} \leq G(s, \eta) D(\eta)
$$


with

$$
G(s, \eta)=\sqrt{2} C\left(\delta_{s} \frac{1-\eta}{\eta} \frac{1+\delta_{s}}{1-\frac{(1-\eta)}{\eta} \delta_{s}}+\delta_{s}\right)=\sqrt{2} C \frac{\delta_{s}}{\eta} \frac{1}{1-\frac{(1-\eta)}{\eta} \delta_{s}}
$$

We clearly have $G(s, \eta) \underset{s \rightarrow \infty}{\longrightarrow} 0$. Since $\delta_{s}$ is convergent, we have $\delta_{s} \leq \delta_{\max }<\infty$. From condition (A.6), we then have $G(s, \eta) \leq \sqrt{2} C \frac{\gamma+\delta_{\max }}{1-\gamma}$, which ends the proof.

Theorem 4 tells us that for any fixed $\eta>0$, the solution $u^{\eta, s}$ of problem $\left(\mathrm{P}^{\eta, s}\right)$ converges towards the solution $u^{\eta}$ of $\left(\mathrm{P}^{\eta}\right)$, in the sense of the norm induced by $A_{\eta}$, which is equivalent to the norm in $\mathcal{W}^{\square}$. This theorem also indicates that when $\eta \rightarrow 0$, the convergence of the sequence $u^{\eta, s}$ may be influenced by the choice of the prolongation $f^{\eta}$ of $f$. A sufficient condition for this convergence not to be influenced by the prolongation is to take

$$
f^{\eta}=\hat{f} \eta^{k}, \quad k \geq 1 / 2, \quad \text { on } \Omega^{\square} \backslash \Omega
$$

with $\hat{f}$ a given prolongation of $f$, such that $\lim _{\eta \rightarrow 0} D(\eta)<\infty$.

\section{B Convergence of separated representations of indicator functions}

We here discuss the assumptions on the indicator function $I$ which are required in order to obtain a uniform convergence of the Karhunen-Loève expansion $I_{s}(x, \xi)=\sum_{i=1}^{s} g_{i}(x) \chi_{i}(\xi)$ (assumption 3), thus making possible the verification of condition (41). We recall that this condition ensures the well-posedness of problems $\left(\mathrm{P}^{\eta, s}\right)$ and the convergence of the associated approximations $u^{\eta, s}$ (see appendix A). More details can be found in [4].

We first suppose that correlation kernel $c_{I}$ is continuous, which is a reasonable assumption that can be verified in practice. Then, from Mercer's theorem [27], we have ${ }^{15}$

$$
\lim _{s \longrightarrow \infty}\left\|I-I_{s}\right\|_{L^{\infty}\left(\Omega^{\square} ; L_{P_{\xi}}^{2}(\Xi)\right)}=0
$$

If in addition, we suppose that

(c1) the images $\left\{\chi_{i}(\Xi)\right\}_{i=1}^{\infty}$ are uniformly bounded in $\mathbb{R}$, i.e. there exists a bounded set $\Upsilon \subset \mathbb{R}$ such that $\chi_{i}(\Xi) \subset \Upsilon$ for all $i \geq 1$,

(c2) the functions $\left\{g_{i}\right\}_{i=1}^{\infty}$ are sufficiently smooth on $\overline{\Omega^{\square}}$ and uniformly bounded, i.e. $\max _{i \geq 1}\left\|g_{i}\right\|_{L^{\infty}\left(\Omega^{\square}\right)}<\infty$,

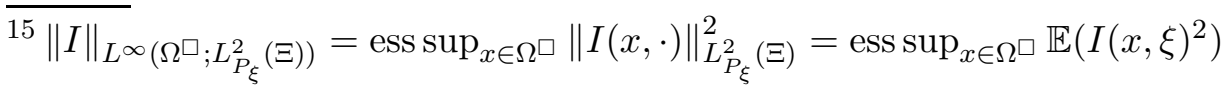


(c3) the eigenvalues of the correlation operator $\gamma_{i}=\left\|g_{i} \chi_{i}\right\|_{L^{2}\left(\Xi \times \Omega^{\square}\right)}^{2}$ have a decay $\gamma_{i}=O\left(\frac{1}{1+i^{q}}\right)$ for some $q>1$,

then we have the uniform convergence of $I_{s}$ towards $I$, i.e.

$$
\lim _{s \longrightarrow \infty}\left\|I-I_{s}\right\|_{L_{P_{\xi}}^{\infty}\left(\Xi ; L^{\infty}\left(\Omega^{\square}\right)\right)}=0
$$

Considering normalized functions $g_{i} \in L^{2}\left(\Omega^{\square}\right)$, we have

$$
\begin{aligned}
\left|\chi_{i}(y)\right| & =\left|\int_{\Omega^{\square}} I(x, y) g_{i}(x) d x\right| \leq \int_{\Omega^{\square}} I(x, y)\left|g_{i}(x)\right| d x \\
& \leq \int_{\Omega^{\square}}\left|g_{i}(x)\right| d x \leq \operatorname{meas}\left(\Omega^{\square}\right)^{1 / 2}\left\|g_{i}\right\|_{L^{2}\left(\Omega^{\square}\right)}<\infty
\end{aligned}
$$

which gives the condition (c1). Condition (c3) could be verified in practice. However, for the present application, condition (c2) is not verified since correlation kernel $c_{I}$ does not have high regularity properties. In fact, this problem can be circumvented by introducing a regularized version of the indicator function $I$, denoted $I^{r}$, with a sufficiently smooth correlation kernel $c_{I^{r}}$. The solution $u^{\eta, r}$ of problem $\left(\mathrm{P}^{\eta}\right)$, with $I$ replaced by $I^{r}$, can be chosen arbitrarily close to the solution $u^{\eta}$. Then, we can define a sequence of problems $\left(\mathrm{P}^{\eta, s}\right)$ associated with separated representations $I_{s}^{r}$ of $I^{r}$. Denoting by $u^{\eta, r, s}$ their solutions, sufficient conditions would be verified in order to guarantee the convergence with $s$ of $u^{\eta, r, s}$ towards $u^{\eta, r}$. The effect of the regularization of $I$ is illustrated in section 4.3.3.

\section{References}

[1] A. Ammar, B. Mokdad, F. Chinesta, and R. Keunings. A new family of solvers for some classes of multidimensional partial differential equations encountered in kinetic theory modelling of complex fluids. Journal of Non-Newtonian Fluid Mechanics, 139(3):153-176, 2006.

[2] M. Arnst and R. Ghanem. Probabilistic Electromechanical Modeling of Nanostructures with Random Geometry. Journal Of Computational And Theoretical Nanoscience, 6(10):2256-2272, 2009.

[3] I. Babuska, U. Banerjee, and J. E. Osborn. Survey of meshless and generalized finite element methods: A unified approach. Acta Numerica, 12:1-125, 2003.

[4] I. Babuska, R. Tempone, and G. E. Zouraris. Solving elliptic boundary value problems with uncertain coefficients by the finite element method: the stochastic formulation. Computer Methods in Applied Mechanics and Engineering, 194:1251-1294, 2005. 
[5] C. Canuto and T. Kozubek. A fictitious domain approach to the numerical solution of pdes in stochastic domains. Numerische Mathematik, 107(2):257293, 2007.

[6] F. Chinesta, A. Ammar, and E. Cueto. Recent advances in the use of the Proper Generalized Decomposition for solving multidimensional models. Archives of Computational Methods in Engineering, 17:327-350, 2010.

[7] A. Doostan and G. Iaccarino. A least-squares approximation of partial differential equations with high-dimensional random inputs. Journal of Computational Physics, 228(12):4332-4345, 2009.

[8] A. Falco and A. Nouy. A Proper Generalized Decomposition for the solution of elliptic problems in abstract form by using a functional Eckart-Young approach. Journal of Mathematical Analysis and Applications, 376:469-480, 2011.

[9] R. Ghanem and P. Spanos. Stochastic finite elements: a spectral approach. Springer, Berlin, 1991.

[10] R. Ghanem and W. Brzakala. Stochastic finite-element analysis of soil layers with random interface. Journal of Engineering Mechanics, 122(4):361-369, 1996.

[11] R. Glowinski, T.W. Pan, R.O. Wells, and X.D. Zhou. Wavelet and finite element solutions for the Neumann problem using fictitious domains. Journal of Computational Physics, 126(1):40-51, JUN 1996.

[12] P. Ladevèze, J.C. Passieux, and D. Néron. The LATIN multiscale computational method and the Proper Generalized Decomposition. Computer Methods in Applied Mechanics and Engineering, 199(21-22):1287-1296, 2010.

[13] O. P. Le Maitre and O. M. Knio. Spectral Methods for Uncertainty Quantification With Applications to Computational Fluid Dynamics. Scientific Computation. 2010.

[14] H. G. Matthies. Stochastic finite elements: Computational approaches to stochastic partial differential equations. Zamm-Zeitschrift Fur Angewandte Mathematik Und Mechanik, 88(11):849-873, 2008.

[15] H. G. Matthies and A. Keese. Galerkin methods for linear and nonlinear elliptic stochastic partial differential equations. Computer Methods in Applied Mechanics and Engineering, 194(12-16):1295-1331, 2005.

[16] P. Surya Mohan, Prasanth B. Nair, and Andy J. Keane. Stochastic projection schemes for deterministic linear elliptic partial differential equations on random domains. Int. J. Numer. Meth. Engng., 85:874-895, 2011.

[17] A. Nouy. A generalized spectral decomposition technique to solve a class of linear stochastic partial differential equations. Computer Methods in Applied Mechanics and Engineering, 196(45-48):4521-4537, 2007. 
[18] A. Nouy. Generalized spectral decomposition method for solving stochastic finite element equations: invariant subspace problem and dedicated algorithms. Computer Methods in Applied Mechanics and Engineering, 197:4718-4736, 2008.

[19] A. Nouy. Recent developments in spectral stochastic methods for the numerical solution of stochastic partial differential equations. Archives of Computational Methods in Engineering, 16(3):251-285, 2009.

[20] A. Nouy. A priori model reduction through proper generalized decomposition for solving time-dependent partial differential equations. Computer Methods in Applied Mechanics and Engineering, 199(23-24):1603-1626, 2010.

[21] A. Nouy. Proper Generalized Decompositions and separated representations for the numerical solution of high dimensional stochastic problems. Archives of Computational Methods in Engineering, 17:403-434, 2010.

[22] A. Nouy and A. Clement. Extended stochastic finite element method for the numerical simulation of heterogenous materials with random material interfaces. Int. J. for Numerical Methods in Engineering, 83(10):127-155, 2010.

[23] A. Nouy, A. Clément, F. Schoefs, and N. Moës. An extended stochastic finite element method for solving stochastic partial differential equations on random domains. Computer Methods in Applied Mechanics and Engineering, 197:4663$4682,2008$.

[24] A. Nouy and A. Falco. Constrained tensor product approximations based on penalized best approximations. submitted. oai:hal.archives-ouvertes.fr:hal00577942

[25] I. Ramière, P. Angot, and M. Belliard. A fictitious domain approach with spread interface for elliptic problems with general boundary conditions. Computer Methods in Applied Mechanics and Engineering, 196(4-6):766 - 781, 2007.

[26] I. Ramière, P. Angot, and M. Belliard. A general fictitious domain method with immersed jumps and multilevel nested structured meshes. Journal of Computational Physics, 225(2):1347 - 1387, 2007.

[27] F. Riesz and B. Sz.-Nagy. Functional Analysis. Dover Publications, New York, NY, 1990.

[28] C. Soize. Non-gaussian positive-definite matrix-valued random fields for elliptic stochastic partial differential operators. Computer Methods in Applied Mechanics and Engineering, 195(1-3):26-64, 2006.

[29] D. M. Tartakovsky and D. Xiu. Stochastic analysis of transport in tubes with rough walls. Journal of Computational Physics, 217:248-259, 2006.

[30] D. Xiu. Fast numerical methods for stochastic computations: a review. Comm. Comput. Phys., 5:242-272, 2009.

[31] D. Xiu and D. M. Tartakovsky. Numerical methods for differential equations in random domains. SIAM J. Sci. Comput., 28(3):1167-1185, 2006. 